ESAIM: PROCEEDINGS AND SURVEYS, March 2015, Vol. 50, p. 144-168

Franck BOYER, Thierry GALLOUET, Raphaèle HERBIN and Florence HUBERT Editors

\title{
PBDW STATE ESTIMATION: NOISY OBSERVATIONS; CONFIGURATION-ADAPTIVE BACKGROUND SPACES; PHYSICAL INTERPRETATIONS
}

\author{
Yvon Maday ${ }^{1}$, Anthony T Patera ${ }^{2}$, James D Penn ${ }^{2}$ And Masayuki Yano ${ }^{2}$
}

\begin{abstract}
We provide extended analyses and interpretations of the parametrized-background data-weak (PBDW) formulation, a real-time in situ data assimilation framework for physical systems modeled by parametrized partial differential equations. The new contributions are threefold. First, we conduct an a priori error analysis for imperfect observations: we provide a bound for the variance of the state error and identify distinct contributions to the noise-induced error. Second, we illustrate the elements of the PBDW formulation for a physical system, a raised-box acoustic resonator, and provide detailed interpretations of the data assimilation results in particular related to model and data contributions. Third, we present and demonstrate an adaptive PBDW formulation in which we incorporate unmodeled physics identified through data assimilation of a select few configurations.

KEYWORDS: variational data assimilation; parametrized partial differential equations; model order reduction; imperfect observations; acoustics.
\end{abstract}

\section{INTRODUCTION}

Numerical prediction based on a given mathematical model is often inadequate due to limitations imposed by available knowledge, calibration requirements, and computational costs. Accurate prediction thus requires the incorporation of experimental observations to accommodate both anticipated, or parametric, uncertainty as well as unanticipated, or nonparametric, uncertainty. Towards this end, we introduced in [16] a Parametrized-Background Data-Weak (PBDW) formulation for variational data assimilation; the formulation combines a "best-knowledge" model encoded in a parametrized partial differential equation (PDE) and experimental observations to provide a real-time in situ estimation of the state of the physical system.

We now state the precise problem that the PBDW formulation addresses. We first denote by $\mathcal{C}$ the configuration of the physical system. In the context of the raised-box acoustic resonator introduced in [16] and revisited in Section 5 of the current paper, $\mathcal{C}$ encodes information which describes the system: operating conditions, such as the frequency; environmental factors, such as the temperature; physical constituents, such as the materials. We now consider a physical system in configuration $\mathcal{C} \in \mathcal{S}$, where $\mathcal{S}$ is the set of all configurations of interest. The PBDW formulation then integrates a parametrized mathematical model and $M$ experimental observations associated with the configuration $\mathcal{C}$ to estimate the true field $u^{\text {true }}[\mathcal{C}]$ as well as any desired output $\ell^{\text {out }}\left(u^{\text {true }}[\mathcal{C}]\right) \in \mathbb{C}$ for given output functional $\ell^{\text {out }}$.

The PBDW formulation is endowed with the following characteristics:

1 Sorbonne Universités, UPMC Univ Paris 06 and CNRS UMR 7598, Laboratoire Jacques-Louis Lions, F-75005, Paris, France; Institut Universitaire de France; Division of Applied Mathematics, Brown University, Providence RI, USA. Email: maday@ann.jussieu.fr

2 Department of Mechanical Engineering, Massachusetts Institute of Technology, 77 Massachusetts Avenue, Cambridge, MA 02139, USA. Email: \{patera, pennjam, myano\}@mit.edu

(c) EDP Sciences, SMAI 2015 
- Weak formulation. We interpret $M$ experimental data as an $M$-dimensional experimentally observable space. We then abstract the estimate based on $M$ observations as a finite-dimensional approximation - effected by projection-by-data — of an infinite-dimensional variational problem.

- Actionable a priori theory. The weak formulation facilitates the construction of a priori error estimates informed by the standard analysis techniques developed for PDEs [17]. The a priori theory guides the optimal choice of the parametrized mathematical model and the experimental observations.

- Background space. The PBDW formulation incorporates background spaces that accommodate anticipated parametric uncertainty. The background space is constructed in two steps: i) the identification of a parametrized PDE that best reflects our (prior) best knowledge of the phenomenon under consideration and the associated anticipated uncertainty - a best-knowledge model —; ii) the application of a model reduction technique - the WeakGreedy algorithm of the certified reduced basis method [18] — to encode the knowledge in the parametrized model in a linear space appropriate for real-time evaluation.

- Design of experiment. The PBDW formulation incorporates the SGreedy algorithm which identifies a quasi-optimal set of observations from a library of experimentally realizable observations in order to maximize the stability of the data assimilation.

- Correction of unmodeled physics. The PBDW formulation, unlike many parameter calibration methods, provides a mechanism by which to correct the unanticipated and nonparametric uncertainty present in the physical model. The unmodeled physics is included through an "update field" that completes the inevitably deficient best-knowledge model. In short, our formulation provides an update field that completes the physics in the model.

- Online computational efficiency. The PBDW formulation provides an offline-online computational decomposition. The online computational cost is $\mathcal{O}(M)$, and thus we may realize real-time state estimation limited only by the speed of the data acquisition.

- Simple implementation and generality. The mathematical model appears only in the offline stage. The formulation permits a simple non-intrusive implementation and the incorporation of high-fidelity, "expensive," mathematical models.

Several of these ingredients have appeared, often separately, in different contexts. The weak formulation in the PBDW formulation, as in many other data assimilation schemes, is built upon least squares $[2,11-$ 13]. For a recent variational analysis of least squares method in functional setting, we refer to Cohen et al. [7]. The best-knowledge background space in PBDW, as opposed to a background singleton element in 3dVAR $[2,12,13]$, is found in earlier work in the context of gappy proper orthogonal decomposition (Gappy POD) [9,20], generalized empirical interpolation method (GEIM) [14,15], and nearfield acoustical holography (NAH) [6,21]; the PBDW emphasis on parametrized PDEs is shared with the GEIM. The SGreedy optimization procedure is related to the E-optimality criteria considered in the design of experiments [10]. Finally, the correction of unmodeled physics through Riesz representation is first introduced in the work of Bennett [1]; the formulation is also closely related to the approximation by radial basis functions [5]. The detailed formulation, analysis, and demonstration of the PBDW framework is presented in [16].

In this work, we extend the previous analysis and demonstration of the PBDW formulation in a number of ways. In particular, the contributions of this work are threefold:

(1) We provide an a priori error analysis in the presence of observation imperfections. Specifically, we consider observation noise which is zero-mean, homoscedastic, and uncorrelated; we then provide an a priori error bound for the variance of the state estimation error.

(2) We provide a detailed interpretation of the PBDW data assimilation results obtained for a particular (interesting) physical system: a raised-box acoustic resonator. Specifically, we analyze two different configurations of the system: a first configuration for which the dominant dynamics of the physical system is well anticipated, the dynamics is captured by the best-knowledge model, and the modeling error is small; a second configuration for which the dominant dynamics of the physical system is poorly anticipated, the dynamics is absent from the best-knowledge model, and the modeling error is large. 
(3) We provide a strategy to update the PBDW based on empirical representation of unmodeled physics obtained through data assimilation for a select few configurations. The strategy improves the experimental efficiency of the data assimilation procedure for all configurations of interest, and is particularly suited in many-query scenarios. We illustrate the approach for the raised-box acoustic resonator.

The paper is organized as follows. In Section 2, we provide a brief overview of the PBDW formulation; for a more elaborate presentation, we refer to [16]. In Section 3, we provide an a priori error analysis of the PBDW state estimation error in the presence of observation errors; we subsequently identify design requirements for the PBDW formulation. In Section 4, we provide practical procedures by which to implement the PBDW formulation. In Section 5, we apply the PBDW formulation to the raised-box acoustic resonator, provide detailed interpretations of the data assimilation results, and present our configuration adaptive approach.

\section{Formulation}

\subsection{Preliminaries}

By way of preliminaries, we introduce the mathematical notations used throughout this paper. We first introduce the standard complex-valued $\ell^{2}\left(\mathbb{C}^{n}\right)$ space, the set of all $n$-tuples of complex numbers, endowed with the Euclidean inner product $(w, v)_{\ell^{2}\left(\mathbb{C}^{n}\right)} \equiv \sum_{i=1}^{n} w_{i} \bar{v}_{i}$ and induced norm $\|w\|_{\ell^{2}\left(\mathbb{C}^{n}\right)} \equiv \sqrt{(w, w)_{\ell^{2}\left(\mathbb{C}^{n}\right)}}$. Here $\bar{a}$ indicates the complex conjugate of $a$. Note that the inner product is linear in the first argument and antilinear in the second argument; we follow this convention for all our inner products. We next introduce the standard complex-valued $L^{2}(\Omega)$ Hilbert space over the domain $\Omega \in \mathbb{R}^{d}$ endowed with an inner product $(w, v)_{L^{2}(\Omega)} \equiv \int_{\Omega} w \bar{v} d x$ and induced norm $\|w\|_{L^{2}(\Omega)} \equiv \sqrt{(w, w)_{L^{2}(\Omega)}} ; L^{2}(\Omega)$ consists of functions $\left\{w \mid\|w\|_{L^{2}(\Omega)}<\infty\right\}$. We then introduce the $H^{1}(\Omega)$ Hilbert space over $\Omega$ endowed with an inner product $(w, v)_{H^{1}(\Omega)} \equiv \int_{\Omega} \nabla w \cdot \nabla \bar{v} d x+\int_{\Omega} w \bar{v} d x$ and induced norm $\|w\|_{H^{1}(\Omega)} \equiv \sqrt{(w, w)_{H^{1}(\Omega)}} ; H^{1}(\Omega)$ consists of functions $\left\{w \mid\|w\|_{H^{1}(\Omega)}<\infty\right\}$. We also introduce the $H_{0}^{1}(\Omega)$ Hilbert space over $\Omega$ endowed with the $H^{1}(\Omega)$ inner product and norm; $H_{0}^{1}(\Omega)$ consists of functions $\left\{w \in H^{1}(\Omega)|w|_{\partial \Omega}=0\right\}$.

We now introduce a space $\mathcal{U}$, a closed subspace of $H^{1}(\Omega)$, endowed with an inner product $(w, v)$ and induced norm $\|w\| \equiv \sqrt{(w, w)} ; \mathcal{U}$ is a Hilbert space when provided with a norm $\|\cdot\|$ which is equivalent to $\|\cdot\|_{H^{1}(\Omega)}$. We assume that $H_{0}^{1}(\Omega) \subset \mathcal{U} \subset H^{1}(\Omega)$. We next introduce the dual space $\mathcal{U}^{\prime}$ and denote the associated duality pairing by $\langle\cdot, \cdot\rangle_{\mathcal{U}^{\prime} \times \mathcal{U}}$; the pairing is linear in the first argument and antilinear in the second argument. The dual space $\mathcal{U}^{\prime}$ is endowed with the norm $\|\ell\|_{\mathcal{U}^{\prime}} \equiv \sup _{w \in \mathcal{U}}|\ell(w)| /\|w\|$ and consists of functionals $\left\{\ell \mid\|\ell\|_{\mathcal{U}^{\prime}}<\infty\right\}$. The operator $U: \mathcal{U} \rightarrow \mathcal{U}^{\prime}$ associated with the inner product satisfies $\langle U w, v\rangle_{\mathcal{U}^{\prime} \times \mathcal{U}}=(w, v) \forall w, v \in \mathcal{U}$. The Riesz operator $R_{\mathcal{U}}: \mathcal{U}^{\prime} \rightarrow \mathcal{U}$ satisfies, for each linear (and not antilinear) functional $\ell \in \mathcal{U}^{\prime},\left(v, R_{\mathcal{U}} \ell\right)=\ell(v), \forall v \in \mathcal{U}$. We finally define, for any closed subspace $\mathcal{Q} \subset \mathcal{U}$, a projection operator $\Pi_{\mathcal{Q}}: \mathcal{U} \rightarrow \mathcal{Q}$ that satisfies, for any $w \in \mathcal{U},\left(\Pi_{\mathcal{Q}} w, v\right)=(w, v) \forall v \in \mathcal{Q}$.

\subsection{PBDW Statement}

As discussed in the introduction, our goal is the estimation of the deterministic state $u^{\text {true }}[\mathcal{C}] \in \mathcal{U}$ of a physical system in configuration $\mathcal{C} \in \mathcal{S}$ based on a parametrized best-knowledge model and $M$ (potentially noisy) observations. Towards this end, we first introduce a sequence of background spaces that reflect our (prior) best knowledge,

$$
\mathcal{Z}_{1} \subset \cdots \subset \mathcal{Z}_{N_{\max }} \subset \cdots \subset \mathcal{U}
$$

here the second ellipsis indicates that we may consider the sequence of length $N_{\text {max }}$ as resulting from a truncation of an infinite sequence. Our goal is to choose the background spaces such that

$$
\lim _{N \rightarrow \infty} \inf _{w \in \mathcal{Z}_{N}}\left\|u^{\text {true }}[\mathcal{C}]-w\right\| \leq \epsilon \mathcal{Z} \quad \forall \mathcal{C} \in \mathcal{S},
$$

for $\epsilon_{\mathcal{Z}}$ an acceptable tolerance. In words, we choose the background spaces such that the most dominant physics that we anticipate to encounter for various system configurations is well represented for a relatively small $N$. Specifically, the background spaces may be constructed through, for instance, the application of a 
model reduction approach to a parametrized PDE, as discussed in Section 4.1; we refer to [16] for various approaches to generate the background space.

We now characterize our data acquisition procedure. Given a system in configuration $\mathcal{C} \in \mathcal{S}$, we assume our observed data $y^{\text {obs }}[\mathcal{C}] \in \mathbb{C}^{M}$ is of the form,

$$
\forall m=1, \ldots, M, \quad y_{m}^{\mathrm{obs}}[\mathcal{C}]=\ell_{m}^{\mathrm{o}}\left(u^{\text {true }}[\mathcal{C}]\right)+e_{m}
$$

here $y_{m}^{\text {obs }}[\mathcal{C}]$ is the value of the $m$-th observation, $\ell_{m}^{\mathrm{o}} \in \mathcal{U}^{\prime}$ is the linear (and not antilinear) functional associated with the $m$-th observation, and $e_{m}$ is the noise associated with the $m$-th observation. The form of the functional depends on the specific transducer used to acquire data. For instance, if the transducer measures a local state value, then we may model the observation as a Gaussian convolution

$$
\ell_{m}^{\mathrm{o}}(v)=\operatorname{Gauss}\left(v ; x_{m}^{\mathrm{c}}, r_{m}\right) \equiv \int_{\Omega}\left\{\left(2 \pi r_{m}^{2}\right)^{-d / 2} \exp \left(-\frac{\left\|x-x_{m}^{\mathrm{c}}\right\|_{\ell^{2}\left(\mathbb{R}^{d}\right)}^{2}}{2 r_{m}^{2}}\right)\right\} v(x) d x
$$

where $x_{m}^{\mathrm{c}} \in \mathbb{R}^{d}$ is the center of the transducer, and $r_{m} \in \mathbb{R}_{>0}$ is the filter width of the transducer; localized observation is of particular interest in this work. Concerning the form of the noises $\left(e_{m}\right)_{m}$, we make the following three assumptions:

(A1) zero mean: $E\left[e_{m}\right]=0, m=1, \ldots, M$;

(A2) homoscedastic: $E\left[e_{m}^{2}\right]=\sigma^{2}, m=1, \ldots, M$;

(A3) uncorrelated: $E\left[e_{m} e_{n}\right]=0, m \neq n$.

Note that we do not assume that the observation error follows any particular distribution (e.g. normal distribution), but only assume that the mean and the covariance of the distribution exist. We note that in practice the mean and covariance of the data acquired is more readily quantifiable than the distribution.

We now introduce a sequence of function spaces associated with our observations. We first associate with each observation functional $\ell_{m}^{o} \in \mathcal{U}^{\prime}$ an observable function,

$$
\forall m=1, \ldots, M, \quad q_{m}=R_{\mathcal{U}} \ell_{m}^{\mathrm{o}}
$$

the Riesz representation of the functional [1]. We then introduce hierarchical observable spaces,

$$
\forall M=1, \ldots, M_{\max }, \ldots, \quad \mathcal{U}_{M}=\operatorname{span}\left\{q_{m}\right\}_{m=1}^{M}
$$

here the second ellipsis indicates that we may consider the sequence of the length $M_{\max }$ as resulting from a truncation of an infinite sequence. We next introduce an observable state $u_{M}^{\text {obs }}[\mathcal{C}] \in \mathcal{U}$ as any function that satisfies

$$
\forall m=1, \ldots, M, \quad \ell_{m}\left(u_{M}^{\mathrm{obs}}[\mathcal{C}]\right)=y_{m}^{\mathrm{obs}}[\mathcal{C}]
$$

We now note from the construction of the observable space, the definition of the observable state, and the definition of Riesz representation, that

$$
\forall m=1, \ldots, M, \quad\left(u_{M}^{\mathrm{obs}}[\mathcal{C}], q_{m}\right)=\left(u_{M}^{\mathrm{obs}}[\mathcal{C}], R_{\mathcal{U}} \ell_{m}^{\mathrm{o}}\right)=\ell_{m}^{\mathrm{o}}\left(u_{M}^{\mathrm{obs}}[\mathcal{C}]\right)=y_{m}^{\mathrm{obs}}[\mathcal{C}]
$$

we may evaluate the inner product of the observable state and a canonical basis function $q_{m} \in \mathcal{U}_{M}$ as the $m$-th observation. More generally, for any $v=\sum_{m=1}^{M} \mathbf{v}_{m} q_{m} \in \mathcal{U}_{M}, \mathbf{v} \in \mathbb{C}^{M}$,

$$
\left(u_{M}^{\mathrm{obs}}[\mathcal{C}], v\right)=\sum_{m=1}^{M} \overline{\mathbf{v}}_{m} y_{m}^{\mathrm{obs}}[\mathcal{C}]
$$

We say that the space $\mathcal{U}_{M}$ is experimentally observable: for the given inner product, $\mathcal{U}_{M}$ comprises precisely the elements of $\mathcal{U}$ whose norm we can evaluate from observations. Note that we introduce $u_{M}^{\text {obs }}[\mathcal{C}]$ to facilitate 
the presentation and variational analysis of the PBDW formulation; however, we do not reconstruct this "intermediate" field during the actual data assimilation process.

We may now state the PBDW estimation statement: given a physical system in configuration $\mathcal{C} \in \mathcal{S}$, find $\left(u_{N, M}^{*}[\mathcal{C}] \in \mathcal{U}, z_{N, M}^{*}[\mathcal{C}] \in \mathcal{Z}_{N}, \eta_{N, M}^{*}[\mathcal{C}] \in \mathcal{U}\right)$ such that

$$
\left(u_{N, M}^{*}[\mathcal{C}], z_{N, M}^{*}[\mathcal{C}], \eta_{N, M}^{*}[\mathcal{C}]\right)=\underset{\substack{u_{N, M} \in \mathcal{U} \\ z_{N, M} \in \mathcal{Z}_{N} \\ \eta_{N, M} \in \mathcal{U}}}{\arg \inf }\left\|\eta_{N, M}\right\|^{2}
$$

subject to

$$
\begin{aligned}
& \left(u_{N, M}, v\right)=\left(\eta_{N, M}, v\right)+\left(z_{N, M}, v\right) \quad \forall v \in \mathcal{U}, \\
& \left(u_{N, M}, \phi\right)=\left(u_{M}^{\mathrm{obs}}[\mathcal{C}], \phi\right) \quad \forall \phi \in \mathcal{U}_{M} .
\end{aligned}
$$

We may readily derive the associated (reduced) Euler-Lagrange equations as a saddle problem [16]: given a physical system in configuration $\mathcal{C} \in \mathcal{S}$, find $\left(\eta_{N, M}^{*}[\mathcal{C}] \in \mathcal{U}_{M}, z_{N, M}^{*}[\mathcal{C}] \in \mathcal{Z}_{N}\right)$ such that

$$
\begin{aligned}
\left(\eta_{N, M}^{*}[\mathcal{C}], q\right)+\left(z_{N, M}^{*}[\mathcal{C}], q\right) & =\left(u_{M}^{\mathrm{obs}}[\mathcal{C}], q\right) \quad \forall q \in \mathcal{U}_{M} \\
\left(\eta_{N, M}^{*}[\mathcal{C}], p\right) & =0 \quad \forall p \in \mathcal{Z}_{N}
\end{aligned}
$$

and set

$$
u_{N, M}^{*}[\mathcal{C}]=\eta_{N, M}^{*}[\mathcal{C}]+z_{N, M}^{*}[\mathcal{C}]
$$

We emphasize that the inner product that appears on the right hand side $-\left(u_{M}^{\text {obs }}[\mathcal{C}], q\right)-$ can be evaluated for any $q \in \mathcal{U}_{M}$ directly from the experimental data $y^{\text {obs }}[\mathcal{C}]$, as described by $(1)$.

We note that the PBDW state estimate $u_{N, M}^{*}[\mathcal{C}]$ in $(4)$ consists of two components: the element in the background space, $z_{N, M}^{*}[\mathcal{C}]$; the element in the observable space, $\eta_{N, M}^{*}[\mathcal{C}]$. The background space $\mathcal{Z}_{N}$ accommodates anticipated uncertainty, and the (experimentally observable) update space $\mathcal{U}_{M}$ accommodates unanticipated uncertainty. Specifically, as evident from the minimization statement (2), we seek the state estimate $u_{N, M}^{*}$ which is consistent with the $M$ observations and which minimizes the update contribution. We note that $\eta_{N, M}^{*}[\mathcal{C}] \in \mathcal{U}_{M}$ but also, from the orthogonality relation $(3)_{2}, \eta_{N, M}^{*}[\mathcal{C}] \in \mathcal{U}_{M} \cap \mathcal{Z}_{N}^{\perp}: \eta_{N, M}^{*}$ thus augments, or complements, an incomplete background space $\mathcal{Z}_{N}$; it is for this reason that we refer to the observable space $\mathcal{U}_{M}$ as the update space.

We make a few remarks about the PBDW saddle (3). First, the saddle problem (3) is well-posed for an appropriate pair of the background space $\mathcal{Z}_{N}$ and the observable space $\mathcal{U}_{M}$; the precise condition required for well-posedness is discussed in Section 3 in the context of an a priori error analysis. Second, there is no reference to any mathematical model in the saddle problem; the connection to the mathematical model is through the hierarchical linear background spaces $\mathcal{Z}_{N}, N=1, \ldots, N_{\max }, \ldots$ The absence of the mathematical model permits application to a wide class of problems, including problems for which the mathematical model is too expensive computationally to invoke in real-time. In addition, the method is perforce non-intrusive, which greatly simplifies the implementation.

\subsection{Algebraic Form: Offline-Online Computational Procedure}

We now introduce an algebraic form of the PBDW formulation that is amenable to computation. Towards this end, we introduce a $\mathcal{N}$-dimensional approximation of the infinite-dimensional space $\mathcal{U}, \mathcal{U}^{\mathcal{N}}$. We similarly introduce approximations of subspaces $\mathcal{Z}_{N} \subset \mathcal{U}$ and $\mathcal{U}_{M} \subset \mathcal{U}, \mathcal{Z}_{N}^{\mathcal{N}} \subset \mathcal{U}^{\mathcal{N}}$ and $\mathcal{U}_{M}^{\mathcal{N}} \subset \mathcal{U}^{\mathcal{N}}$, respectively. The space $\mathcal{U}^{\mathcal{N}}$ typically arises from a finite-element discretization; we assume that $\cup_{\mathcal{N}} \mathcal{U}^{\mathcal{N}}$ is dense in $\mathcal{U}$.

We then introduce a basis for the background space $\left\{\zeta_{n} \in \mathcal{U}^{\mathcal{N}}\right\}_{n=1}^{N}$. The background space is thus given by $\mathcal{Z}_{N}^{\mathcal{N}} \equiv \operatorname{span}\left\{\zeta_{n}\right\}_{n=1}^{N}$. The associated background space operator is $Z: \mathbb{C}^{N} \rightarrow \mathcal{Z}_{N}^{\mathcal{N}}$ such that

$$
Z \mathbf{z}=\sum_{n=1}^{N} \mathbf{z}_{n} \zeta_{n} \quad \text { in } \mathcal{U}^{\mathcal{N}}
$$


we may represent any $z \in \mathcal{Z}_{N}^{\mathcal{N}}$ as $z=Z \mathbf{z}$ for the matrix $Z \in \mathbb{C}^{\mathcal{N} \times N}$ and for some $\mathbf{z} \in \mathbb{C}^{N}$.

Similarly, for the observation functionals $\left\{\ell_{m}^{o}\right\}_{m=1}^{M}$, we introduce the associated observation operator $L: \mathcal{U}^{\mathcal{N}} \rightarrow \mathbb{C}^{M}$ such that

$$
\forall m=1, \ldots, M, \quad(L w)_{m}=\ell_{m}^{\circ}(w) \quad \forall w \in \mathcal{U}^{\mathcal{N}}
$$

given an algebraic (vector) representation of the observation functional $\ell_{m}^{\circ}: \mathcal{U}^{\mathcal{N}} \rightarrow \mathbb{C}$, the observation operator $L$ is a matrix $L \in \mathbb{C}^{M \times \mathcal{N}}$ that arises from the concatenation of the vectors. We then introduce the canonical basis for the update space $\left\{q_{m} \equiv R_{\mathcal{U}}^{\mathcal{N}} \ell_{m}^{\circ}\right\}_{m=1}^{M}$; here $R_{\mathcal{U}}^{\mathcal{N}} \ell_{m}^{\circ} \in \mathcal{U}^{\mathcal{N}}$ satisfies $\left(v, R_{\mathcal{U}}^{\mathcal{N}} \ell_{m}^{\circ}\right)=\ell_{m}^{\circ}(v)$, $\forall v \in \mathcal{U}^{\mathcal{N}}$. The update space is given by $\mathcal{U}_{M}^{\mathcal{N}} \equiv \operatorname{span}\left\{q_{m}\right\}_{m=1}^{M}$. The associated update space operator is $Q: \mathbb{C}^{M} \rightarrow \mathcal{U}_{M}^{\mathcal{N}}$ such that

$$
Q \mathbf{v}=\sum_{m=1}^{M} \mathbf{v}_{m} q_{m} \quad \text { in } \mathcal{U}^{\mathcal{N}}, \quad \forall \mathbf{v} \in \mathbb{C}^{M}
$$

we may represent any $v \in \mathcal{U}_{M}^{\mathcal{N}}$ as $v=Q \mathbf{v}$ for the matrix $Q \in \mathbb{C}^{\mathcal{N} \times M}$ and some $\mathbf{v} \in \mathbb{C}^{M}$.

We now state the algebraic form of the PBDW saddle problem (3) such that $\eta_{N, M}^{*}[\mathcal{C}]=Q \boldsymbol{\eta}^{*}[\mathcal{C}] \in \mathcal{U}_{M}^{\mathcal{N}}$ and $z_{N, M}^{*}[\mathcal{C}]=Z \mathbf{z}^{*}[\mathcal{C}] \in \mathcal{Z}_{N}^{\mathcal{N}}$ : given observation data $y^{\text {obs }}[\mathcal{C}] \in \mathbb{C}^{M}$ associated with a physical system in configuration $\mathcal{C} \in \mathcal{S}$, find $\left(\boldsymbol{\eta}^{*}[\mathcal{C}] \in \mathbb{C}^{M}, \mathbf{z}^{*}[\mathcal{C}] \in \mathbb{C}^{N}\right)$ such that

$$
\left(\begin{array}{cc}
\mathbf{A} & \mathbf{B} \\
\mathbf{B}^{H} & 0
\end{array}\right)\left(\begin{array}{l}
\boldsymbol{\eta}^{*}[\mathcal{C}] \\
\mathbf{z}^{*}[\mathcal{C}]
\end{array}\right)=\left(\begin{array}{c}
y^{\mathrm{obs}}[\mathcal{C}] \\
0
\end{array}\right)
$$

where

$$
\begin{aligned}
& \mathbf{A} \equiv Q^{\dagger} U Q=L Q \in \mathbb{C}^{M \times M} \\
& \mathbf{B} \equiv Q^{\dagger} U Z=L Z \in \mathbb{C}^{M \times N}
\end{aligned}
$$

where $Q^{\dagger}:\left(\mathcal{U}_{M}^{\mathcal{N}}\right)^{\prime} \rightarrow \mathbb{C}^{M}$ is the adjoint of $Q: \mathbb{C}^{M} \rightarrow \mathcal{U}_{M}^{\mathcal{N}}$.

The offline-online decomposition is clear from the construction. In the offline stage, we compute the supermatrices $\mathbf{A}_{\max } \equiv L_{\max } Q_{\max } \in \mathbb{C}^{M_{\max } \times M_{\max }}$ and $\mathbf{B}_{\max } \equiv L_{\max } Z_{\max } \in \mathbb{C}^{M_{\max } \times N_{\max }}$, where $L_{\max } \in$ $\mathbb{C}^{M_{\max } \times \mathcal{N}}$ is the observation operator associated with the $M_{\max }$ observations, $Z_{\max } \in \mathbb{C}^{\mathcal{N} \times N_{\max }}$ is the background operator associated with the $N_{\max }$-dimensional background space, and $Q_{\max } \in \mathbb{C}^{\mathcal{N} \times M}$ is the update operator associated with the $M_{\max }$-dimensional update space. In the online stage, we first extract the principle submatrices of $\mathbf{A}_{\max } \in \mathbb{C}^{M_{\max } \times M_{\max }}$ and $\mathbf{B}_{\max } \in \mathbb{C}^{M_{\max } \times N_{\max }}$ to form $\mathbf{A} \in \mathbb{C}^{M \times M}$ and $\mathbf{B} \in \mathbb{C}^{M \times N}$, respectively; we then solve the saddle system (5).

\section{A Priori ERror Analysis}

\subsection{Error Decomposition}

We now analyze the error associated with the PBDW data assimilation procedure. We attribute our error to two distinct contributions. The first contribution arises from the fact that modeling error is inevitable (i.e. $u^{\text {true }} \notin \mathcal{Z}_{N}$ ) and that the update space $\mathcal{U}_{M}$ with which we augment $\mathcal{Z}_{N}$ is finite dimensional; this error is present even if each observation is noise-free - that is, even if we could probe the true deterministic state - and hence we refer to this component of the error as the deterministic error. The second contribution arises from the fact that each observation is noisy, corrupted by the random observation noise; we refer to this component of the error as the stochastic error.

In order to precisely distinguish the two components of the error, we introduce the PBDW state estimate that would be obtained in the absence of the noise $e, u_{N, M}^{\text {nf }}[\mathcal{C}] \in \mathcal{U}$; the superscript "nf" denotes "noise-free." The noise-free state is governed by the following weak statement: given a physical system in configuration 
$\mathcal{C} \in \mathcal{S}$, find $\left(\eta_{N, M}^{\mathrm{nf}}[\mathcal{C}] \in \mathcal{U}_{M}, z_{N, M}^{\mathrm{nf}}[\mathcal{C}] \in \mathcal{Z}_{N}\right)$ such that

$$
\begin{aligned}
\left(\eta_{N, M}^{\mathrm{nf}}[\mathcal{C}], q\right)+\left(z_{N, M}^{\mathrm{nf}}[\mathcal{C}], q\right) & =\left(u^{\text {true }}[\mathcal{C}], q\right) \quad \forall q \in \mathcal{U}_{M}, \\
\left(\eta_{N, M}^{\mathrm{nf}}[\mathcal{C}], p\right) & =0 \quad \forall p \in \mathcal{Z}_{N},
\end{aligned}
$$

and set

$$
u_{N, M}^{\mathrm{nf}}[\mathcal{C}]=\eta_{N, M}^{\mathrm{nf}}[\mathcal{C}]+z_{N, M}^{\mathrm{nf}}[\mathcal{C}]
$$

Note that the saddle problem is identical to the PBDW saddle (3) except that the right hand side of the first equation is $\left(u^{\text {true }}[\mathcal{C}], q\right)$ and hence we probe the true deterministic state.

The following lemma provides a characterization of the error in terms of the deterministic and stochastic components.

Lemma 1. The expectation of the norm of the state error may be decomposed into deterministic and stochastic components and is bounded by

$$
E\left[\left\|u^{\text {true }}[\mathcal{C}]-u_{N, M}^{*}[\mathcal{C}]\right\|\right] \leq\left\|u^{\text {true }}[\mathcal{C}]-u_{N, M}^{\mathrm{nf}}[\mathcal{C}]\right\|+E\left[\left\|u_{N, M}^{\mathrm{nf}}[\mathcal{C}]-u_{N, M}^{*}[\mathcal{C}]\right\|\right]
$$

here $u^{\text {true }}[\mathcal{C}]$ is the true deterministic state, $u_{N, M}^{*}[\mathcal{C}]$ is the PBDW estimate given by $(3), u_{N, M}^{\mathrm{nf}}[\mathcal{C}]$ is the noise-free estimate given by (6), and $E$ refers to expectation.

Proof. The result follows from the triangle inequality and the fact that both $u^{\text {true }}[\mathcal{C}]$ and $u_{N, M}^{\mathrm{nf}}[\mathcal{C}]$ are deterministic.

\subsection{Error Analysis and Interpretation: Deterministic Component}

We first bound the deterministic error.

Proposition 2. The deterministic component of the error is bounded by

$$
\left\|u^{\text {true }}[\mathcal{C}]-u_{N, M}^{\mathrm{nf}}[\mathcal{C}]\right\| \leq\left(1+\frac{1}{\beta_{N, M}}\right) \inf _{q \in \mathcal{U}_{M} \cap \mathcal{Z}_{\bar{N}}^{\perp}}\left\|\Pi_{\mathcal{Z}_{\bar{N}}} u^{\text {true }}[\mathcal{C}]-q\right\|
$$

where $\beta_{N, M}$ is the inf-sup constant given by

$$
\beta_{N, M} \equiv \inf _{w \in \mathcal{Z}_{N}} \sup _{v \in \mathcal{U}_{M}} \frac{(w, v)}{\|w\|\|v\|}
$$

here $u^{\text {true }}[\mathcal{C}]$ is the true deterministic state, and $u_{N, M}^{\mathrm{nf}}[\mathcal{C}]$ is the noise-free estimate given by $(6)$.

Proof. See [16], Proposition 2.

We identify three contributions to the deterministic error bound (7). First is the inf-sup (or stability) constant, $\beta_{N, M} \in[0,1]$, which is a metric of how well the elements in the background space are observed and are distinguished by the observable space $\mathcal{U}_{M}$. Note that the stability constant is a decreasing function of the dimension of the background space, $N$, and an increasing function of the dimension of the observable space, $M$.

The second contribution to the deterministic error bound is the background best-fit error, $\epsilon_{N}^{\text {bk }}\left(u^{\text {true }}[\mathcal{C}]\right) \equiv$ $\inf _{z \in \mathcal{Z}_{N}}\left\|u^{\text {true }}[\mathcal{C}]-z\right\| \equiv\left\|\Pi_{\mathcal{Z}_{N}} u^{\text {true }}[\mathcal{C}]\right\|$, which is a non-increasing function of the dimension of the background space, $N$. We recall that we strive to select the background spaces $\mathcal{Z}_{N}, N=1, \ldots, N_{\max }$, such that this best-fit error is small for a relatively small $N$.

The third contribution to the deterministic error bound is the update best-fit error, $\inf _{q \in \mathcal{U}_{M} \cap \mathcal{Z}_{N}^{\perp}} \| \Pi_{\mathcal{Z}_{N}^{\perp}} u^{\text {true }}$ $q \|$. The error is a non-increasing function of the dimension of the update space, $M$. The error decreases as the update space enriches and corrects for more and more components of $u^{\text {true }}[\mathcal{C}]$ that lie outside of the inevitably deficient background space $\mathcal{Z}_{N}$. We in particular note that, for a sequence $\mathcal{U}_{M} \rightarrow \mathcal{U}$ as $M \rightarrow \infty$, 
we expect the deterministic component of the error to vanish as $M \rightarrow \infty$; in other words, the noise-free estimate $u_{N, M}^{\mathrm{nf}}[\mathcal{C}]$ converges to $u^{\text {true }}[\mathcal{C}]$ as $M \rightarrow \infty$. However, this convergence in $M$ is unfortunately rather slow: in $d$ physical dimensions, the error may converge as slowly as $M^{-1 / d}[5,16]$.

\subsection{Error Analysis and Interpretation: Stochastic Component}

We now analyze the stochastic component of the error.

Proposition 3. Suppose the observation error e satisfies the assumptions (A1), (A2), and (A3). Then, the mean of the stochastic error is zero:

$$
E\left[u_{N, M}^{\mathrm{nf}}[\mathcal{C}]-u_{N, M}^{*}[\mathcal{C}]\right]=0
$$

here $u_{N, M}^{\mathrm{nf}}[\mathcal{C}]$ is the noise-free estimate given by (6), and $u_{N, M}^{*}[\mathcal{C}]$ is the $P B D W$ estimate given by (3). Moreover, the variance of the stochastic error is bounded by

$$
\sqrt{E\left[\left\|u_{N, M}^{\mathrm{nf}}[\mathcal{C}]-u_{N, M}^{*}[\mathcal{C}]\right\|^{2}\right]} \leq \sigma\left(1+\frac{2}{\beta_{N, M}^{2}}\right) \sqrt{\operatorname{trace}\left(\mathbf{A}^{-1}\right)}
$$

where $\mathbf{A} \equiv Q^{\dagger} U Q \in \mathbb{C}^{M \times M}, \beta_{N, M}$ is the inf-sup constant defined in (8), and $\sigma^{2}$ is the variance of the measurement noise.

Proof. For notational simplicity, we suppress in this proof the configuration parameter $[\mathcal{C}]$ for all state and state estimates.

We first define $\eta_{N, M}^{\mathrm{st}} \equiv \eta_{N, M}^{\mathrm{nf}}-\eta_{N, M}^{*}$ and $z_{N, M}^{\mathrm{st}} \equiv z_{N, M}^{\mathrm{nf}}-z_{N, M}^{*}$. We next subtract (3) from (6) to obtain

$$
\begin{array}{ll}
\left(\eta_{N, M}^{\text {st }}, q\right)+\left(z_{N, M}^{\text {st }}, q\right) & =\left(u^{\text {true }}-u_{M}^{\text {obs }}, q\right) \quad \forall q \in \mathcal{U}_{M}, \\
\left(\eta_{N, M}^{\text {st }}, p\right) & =0 \quad \forall p \in \mathcal{Z}_{N} .
\end{array}
$$

We may express any $q \in \mathcal{U}_{M}$ as $q=\sum_{m=1}^{M} q_{m} \boldsymbol{\alpha}_{m}$, where $q_{m} \equiv R_{\mathcal{U}} \ell_{m}$; hence it follows that the right hand side of $(11)_{1}$ can be expressed as

$$
\left(u^{\text {true }}-u_{M}^{\mathrm{obs}}, q\right)=\sum_{m=1}^{M} \overline{\boldsymbol{\alpha}}_{m}\left(u^{\text {true }}-u_{M}^{\mathrm{obs}}, q_{m}\right)=\sum_{m=1}^{M} \overline{\boldsymbol{\alpha}}_{m}\left(\ell_{m}\left(u^{\text {true }}\right)-\ell_{m}\left(u^{\text {true }}\right)-e_{m}\right)=-\sum_{m=1}^{M} \overline{\boldsymbol{\alpha}}_{m} e_{m} .
$$

Because (11) is linear, the right hand side depends linearly on $e$, and $E[e]=0$, we conclude that $E\left[u_{N, M}^{\mathrm{nf}}-\right.$ $\left.u_{N, M}^{*}\right]=E\left[\eta_{N, M}^{\mathrm{st}}\right]+E\left[z_{N, M}^{\mathrm{st}}\right]=0$; this proves $(9)$.

We next bound the expectation of the norm of the error. We test (11) $)_{1}$ against $\eta_{N, M}^{\text {st }} \in \mathcal{U}_{M} \cap \mathcal{Z}_{N}^{\perp}$ and note that $\left(z_{N, M}^{\mathrm{st}}, \eta_{N, M}^{\mathrm{st}}\right)=0$ to obtain

$$
\left(\eta_{N, M}^{\mathrm{st}}, \eta_{N, M}^{\mathrm{st}}\right)=\left(u^{\mathrm{true}}-u_{M}^{\mathrm{obs}}, \eta_{N, M}^{\mathrm{st}}\right)=\left(\Pi_{\mathcal{U}_{M}}\left(u^{\mathrm{true}}-u_{M}^{\mathrm{obs}}\right), \eta_{N, M}^{\mathrm{st}}\right) .
$$

We now invoke the Cauchy-Schwarz inequality to obtain

$$
\left\|\eta_{N, M}^{\mathrm{st}}\right\| \leq\left\|\Pi_{\mathcal{U}_{M}}\left(u^{\mathrm{true}}-u_{M}^{\mathrm{obs}}\right)\right\|
$$

We in addition note that

$$
\begin{aligned}
& \beta_{N, M}\left\|z^{\mathrm{st}}\right\| \leq \sup _{q \in \mathcal{U}_{M}} \frac{\left(z_{N, M}^{\mathrm{st}}, q\right)}{\|q\|}=\sup _{q \in \mathcal{U}_{M}} \frac{\left(u^{\text {true }}-u_{M}^{\mathrm{obs}}, q\right)-\left(\eta_{N, M}^{\mathrm{st}}, q\right)}{\|q\|} \\
& \leq\left\|\Pi_{\mathcal{U}_{M}}\left(u^{\text {true }}-u_{M}^{\mathrm{obs}}\right)\right\|+\left\|\eta_{N, M}^{\mathrm{st}}\right\| \leq 2\left\|\Pi_{\mathcal{U}_{M}}\left(u^{\text {true }}-u_{M}^{\mathrm{obs}}\right)\right\| ; \\
& 151
\end{aligned}
$$


here the first relation follows from the definition of the inf-sup constant (8), the second relation follows from $(11)_{1}$, the third relation follows from Cauchy-Schwarz, and the last relation follows from (12). We hence obtain an intermediate result,

$$
\left\|u_{N, M}^{\mathrm{nf}}-u_{N, M}^{*}\right\| \leq\left\|\eta_{N, M}^{\mathrm{st}}\right\|+\left\|z_{N, M}^{\mathrm{st}}\right\| \leq\left(1+\frac{2}{\beta_{N, M}}\right)\left\|\Pi_{\mathcal{U}_{M}}\left(u^{\text {true }}-u_{M}^{\mathrm{obs}}\right)\right\|
$$

where the first inequality follows from the triangle inequality.

We now derive an explicit expression for $\Pi_{\mathcal{U}_{M}}\left(u^{\text {true }}-u_{M}^{\text {obs }}\right)$ and then bound its norm. Towards this end, we note that $\Pi_{\mathcal{U}_{M}}\left(u^{\text {true }}-u_{M}^{\text {obs }}\right)=\sum_{m=1}^{M} q_{m} \boldsymbol{\alpha}_{m}$ for $\boldsymbol{\alpha} \in \mathbb{C}^{M}$ that satisfies

$$
\forall m^{\prime}=1, \ldots, M, \quad \sum_{m=1}^{M}\left(q_{m}, q_{m^{\prime}}\right) \boldsymbol{\alpha}_{m}=\left(u^{\text {true }}-u_{M}^{\text {obs }}, q_{m^{\prime}}\right)=-e_{m^{\prime}} .
$$

Recalling $\left(q_{m}, q_{m^{\prime}}\right)=\mathbf{A}_{m^{\prime} m}$, we obtain $\boldsymbol{\alpha}=-\mathbf{A}^{-1} e$. It follows that $\Pi_{\mathcal{U}_{M}}\left(u^{\text {true }}-u_{M}^{\text {obs }}\right)=-Q \mathbf{A}^{-1} e$. Thus, we obtain an equality for the norm,

$$
\left\|\Pi_{\mathcal{U}_{M}}\left(u^{\text {true }}-u_{M}^{\mathrm{obs}}\right)\right\|^{2}=\left\|Q \mathbf{A}^{-1} e\right\|^{2}=e^{H} \mathbf{A}^{-1} e .
$$

We then note that $E\left[e e^{H}\right]=\sigma^{2} \mathbf{I}_{M}$, where $\mathbf{I}_{M} \in \mathbb{C}^{M \times M}$ is the identity matrix, to obtain

$$
E\left[\left\|\Pi_{\mathcal{U}_{M}}\left(u^{\text {true }}-u_{M}^{\text {obs }}\right)\right\|^{2}\right]=E\left[e^{H} \mathbf{A}^{-1} e\right]=E\left[\operatorname{trace}\left(e e^{H} \mathbf{A}^{-1}\right)\right]=\sigma^{2} \operatorname{trace}\left(\mathbf{A}^{-1}\right) .
$$

We finally substitute (15) into (13) to obtain

$$
E\left[\left\|u_{N, M}^{\mathrm{nf}}-u_{N, M}^{*}\right\|^{2}\right] \leq\left(1+\frac{2}{\beta_{N, M}}\right)^{2} \sigma^{2} \operatorname{trace}\left(\mathbf{A}^{-1}\right),
$$

which is the desired bound on the norm of the stochastic error (10).

Remark 4. We may obtain an alternative bound that does not rely on the assumption of uncorrelated noise. At (14), we invoke

$$
\left\|\Pi_{\mathcal{U}_{M}}\left(u^{\text {true }}-u_{M}^{\text {obs }}\right)\right\|^{2}=e^{H} \mathbf{A}^{-1} e \leq \lambda_{\max }\left(\mathbf{A}^{-1}\right)\|e\|_{\ell^{2}\left(\mathbb{C}^{M}\right)} ;
$$

we then take the expectation to obtain

$$
E\left[\left\|\Pi_{\mathcal{U}_{M}}\left(u^{\text {true }}-u_{M}^{\text {obs }}\right)\right\|^{2}\right] \leq \lambda_{\max }\left(\mathbf{A}^{-1}\right) E\left[\|e\|_{\ell^{2}\left(\mathbb{C}^{M}\right)}\right]=\lambda_{\max }\left(\mathbf{A}^{-1}\right) E\left[\sum_{m=1}^{M} e_{m}^{2}\right]=\lambda_{\max }\left(\mathbf{A}^{-1}\right) \sigma^{2} M,
$$

where $\lambda_{\max }\left(\mathbf{A}^{-1}\right)$ it the maximum eigenvalue of the Hermitian matrix $\mathbf{A}^{-1}$, and $\sigma^{2}$ is the variance of the noise. The bound on the norm of the stochastic error is hence

$$
E\left[\left\|u_{N, M}^{\mathrm{nf}}-u_{N, M}^{*}\right\|^{2}\right] \leq\left(1+\frac{2}{\beta_{N, M}}\right)^{2} \sigma^{2} M \lambda_{\max }\left(\mathbf{A}^{-1}\right) .
$$

Note that this bound cannot be sharper than (10) but is valid for correlated noise.

We identify three distinct contributions to the stochastic error bound (10). First is the variance of the observation noise, $\sigma^{2}$; the smaller the variance in the observation noise, the smaller the stochastic error in the state estimate. Note that, while the observation procedure employed dictates the variance of the observation noise $\sigma^{2}$ associated with a single observation, we may reduce the effective variance through 


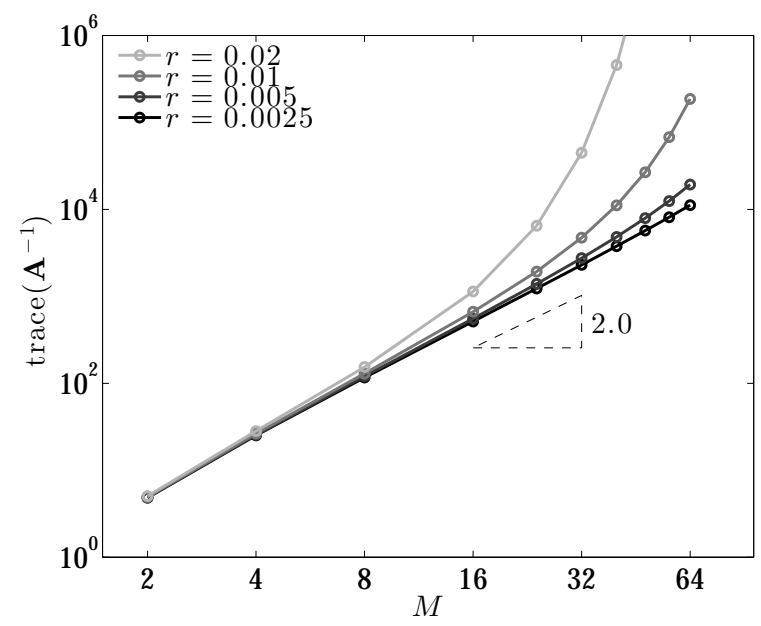

(a) one-dimensional case

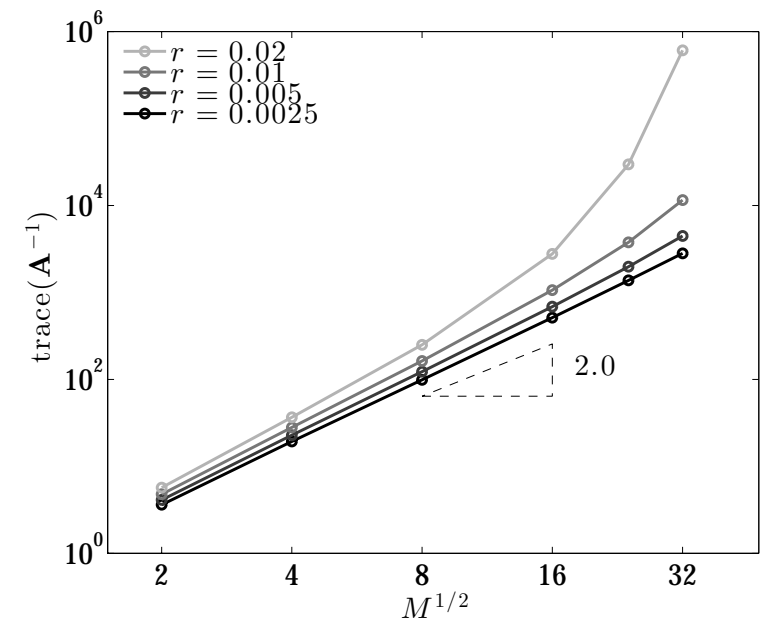

(b) two-dimensional case

FiguRE 1. The scaling of trace $\left(\mathbf{A}^{-1}\right)$ with the dimension of the observable space $\mathcal{U}_{M}$ in (a) one physical dimension and (b) two physical dimensions. The observation functionals are of the form $\ell_{m}^{\circ} \equiv \operatorname{Gauss}\left(\cdot ; x_{m}^{\mathrm{c}}, r\right)$ for uniformly distributed observation centers $\left\{x_{m}^{\mathrm{c}}\right\}_{m=1}^{M}$ and the spread parameter as indicated in the legend.

repeated observations assuming the assumptions (A1) and (A3) are satisfied. In particular, the effective variance of independent, homoscedastic noise scales as $1 / \widetilde{M}$, where $\widetilde{M}$ is the number of repetitions.

The second contribution to the stochastic error is the inf-sup (or stability) constant, $\beta_{N, M}$. The inf-sup constant characterizes the influence of the observation noise on the background component of the stochastic error. As we have discussed in the context of the bound for the deterministic error, the inf-sup constant is a metric of how well the elements in the background space are observed and distinguished by the observable space $\mathcal{U}_{M}$. We can hence maximize the constant through a judicious choice of the observation functionals.

The third contribution to the stochastic error is trace $\left(\mathbf{A}^{-1}\right)$. We in particular wish to understand the relationship between the observable space $\mathcal{U}_{M}$ and $\operatorname{trace}\left(\mathbf{A}^{-1}\right)$. Unfortunately, we have not been able to analyze the spectrum theoretically. Here we empirically study the trace through computation.

We first consider a one-dimensional problem over the unit-segment domain, $\Omega \equiv(0,1)$. The space $\mathcal{U}$ consists of $H^{1}(\Omega)$ functions and is endowed with the standard $H^{1}$ inner product and norm. We introduce Gaussian observation functionals of the form $\ell_{m}^{\mathrm{o}} \equiv \operatorname{Gauss}\left(\cdot ; x_{m}^{\mathrm{c}}, r\right), m=1, \ldots, M$; here we consider equidistributed observation centers $\left\{x_{m}^{c}\right\}_{m=1}^{M}$ over the unit segment and four different values of the spread parameter $r:\{0.0025,0.005,0.01,0.02\}$. The problem is discretized using $128 \mathbb{P}^{8}$ finite elements. The result of the computation is shown in Figure 1(a). We empirically observe that, if the spread parameter $r$ is sufficiently small relative to the spacing between the observation centers $(1 / M)$, then trace $\left(\mathbf{A}^{-1}\right)$ scales algebraically and in particular as $M^{2}$. On the other hand, if the spread parameter $r$ is comparable to the spacing between the observation centers $(1 / M)$, then trace $\left(\mathbf{A}^{-1}\right)$ scales exponentially with $M$.

We next consider a two-dimensional problem over the unit-square domain, $\Omega \equiv(0,1)^{2}$. The space $\mathcal{U}$ consists of $H^{1}(\Omega)$ functions and is endowed with the standard $H^{1}$ inner product and norm. We introduce Gaussian observation functionals of the form $\ell_{m}^{o} \equiv \operatorname{Gauss}\left(\cdot ; x_{m}^{c}, r\right), m=1, \ldots, M$; here we consider equidistributed observation centers $\left\{x_{m}^{\mathrm{c}}\right\}_{m=1}^{M}$ over the unit square and four different values of the spread parameter $r:\{0.0025,0.005,0.01,0.02\}$. The problem is discretized using $64 \times 64 \times 2 \mathbb{P}^{5}$ triangular finite elements. The result of the computation is shown in Figure 1(b). We empirically observe a behavior similar to the one-dimensional case. If the spread parameter $r$ is sufficiently small relative to the spacing between the observation centers $\left(1 / M^{1 / 2}\right)$, then trace $\left(\mathbf{A}^{-1}\right)$ scales algebraically and in particular as $\left(M^{1 / 2}\right)^{2}=M$. If the spread parameter $r$ is comparable to the spacing between the observation centers $\left(1 / M^{1 / 2}\right)$, then trace $\left(\mathbf{A}^{-1}\right)$ scales exponentially with $M$. 
Based on the empirical results for the one- and two-dimensional cases, we speculate that in $d$ dimensions trace $\left(\mathbf{A}^{-1}\right)$ scales as $\left(M^{1 / d}\right)^{2}=M^{2 / d}$ for uniformly distributed observation functionals with sufficiently small spread parameters. From the stochastic error bound (10), we conclude that $\sigma^{2}$ must decrease faster than $M^{-2 / d}$; in other words, since $\sigma^{2} \sim \widetilde{M}^{-1}$ for $\widetilde{M}$ the number of repeated observations, $\widetilde{M}>M^{2 / d}$ to ensure convergence as $M \rightarrow \infty$.

\subsection{Roles of the Background Space $\mathcal{Z}_{N}$ and the Update Space $\mathcal{U}_{M}$}

We now identify the roles played by the background space $\mathcal{Z}_{N}$ and the update space $\mathcal{U}_{M}$ based on the $a$ priori error analyses. We first consider the role of the background space $\mathcal{Z}_{N}$. Proposition 2 shows that, in order to minimize the deterministic error, the space $\mathcal{Z}_{N}$ must provide

$$
\left(\mathrm{R}_{\mathcal{Z}_{N}} 1\right) \text { primary approximation: } \quad \epsilon_{N}^{\mathrm{bk}}\left(u^{\text {true }}[\mathcal{C}]\right) \equiv \inf _{z \in \mathcal{Z}_{N}}\left\|u^{\text {true }}[\mathcal{C}]-z\right\| \quad \text { is small } \forall \mathcal{C} \in \mathcal{S} .
$$

In words, the $\mathcal{Z}_{N}$ must well represent the true state $u^{\text {true }}[\mathcal{C}]$ for all $\mathcal{C} \in \mathcal{S}$ for ideally a relatively small $N$. The background space also influences the stability constant $\beta_{N, M}$ in the deterministic error bound (7) and the stochastic error bound (10); however, we choose to select $\mathcal{Z}_{N}$ solely based on the requirement $\left(\mathrm{R}_{\mathcal{Z}} 1\right)$ and to employ the update space to control the stability constant $\beta_{N, M}$.

We next consider the roles of the update space $\mathcal{U}_{M}$. Proposition 2 shows that, in order to minimize the deterministic error, the space $\mathcal{U}_{M}$ must provide

$$
\begin{aligned}
& \left(\mathrm{R}_{\mathcal{U}_{M}} 1\right) \text { primary stability: } \quad \beta_{N, M} \equiv \inf _{w \in \mathcal{Z}_{N}} \sup _{v \in \mathcal{U}_{M}} \frac{(w, v)}{\|w\|\|v\|}>0, \\
& \left(\mathrm{R}_{\mathcal{U}_{M}} 2\right) \text { secondary approximation: } \\
& \inf _{\eta \in \mathcal{U}_{M} \cap \mathcal{Z}_{N}^{\perp}}\left\|\Pi_{\mathcal{Z}_{\perp}^{\perp}} u^{\text {true }}[\mathcal{C}]-\eta\right\| .
\end{aligned}
$$

In words, $\mathcal{U}_{M}$ must ensure that $\left(\mathrm{R}_{\mathcal{U}_{M}} 1\right)$ the elements in the background space are experimentally observable and distinguishable and $\left(\mathrm{R}_{\mathcal{U}_{M}} 2\right)$ the update space well approximates the functions not in the background space $\mathcal{Z}_{N}$ as to complete any deficiency in $\mathcal{Z}_{N}$. In addition, Proposition 3 shows that, in order to control the stochastic error, the space $\mathcal{U}_{M}$ must also provide

$$
\left(\mathrm{R}_{\mathcal{U}_{M}} 3\right) \text { well-conditioned observations: } \quad \operatorname{trace}\left(\mathbf{A}^{-1}\right) \text { is not large. }
$$

In words, the canonical basis $\left\{q_{m} \equiv R_{\mathcal{U}} \ell_{m}^{o}\right\}_{m=1}^{M}$ of $\mathcal{U}_{M}$ must be well-conditioned in the sense that the eigenvalues of the Gramian matrix $\mathbf{A} \equiv Q^{\dagger} U Q$ are not small. As observed in Section 3.3, for local observations modeled by $\ell_{m}^{\circ} \equiv \operatorname{Gauss}\left(\cdot ; x_{m}^{\mathrm{c}}, r_{m}\right)$, the requirement $\left(\mathrm{R}_{\mathcal{U}_{M}} 3\right)$ is satisfied if the observation centers are sufficiently distinct in the sense that the minimum distance between two observation centers is sufficiently large relative to the filtering width $r_{m}$.

\section{Construction of Spaces}

\subsection{Background Spaces}

As discussed in Section 3.4, we wish to choose a background space that provides $\left(\mathrm{R}_{\mathcal{Z}_{N}} 1\right)$ : the background best-fit error $\epsilon_{N}^{\text {bk }}\left(u^{\text {true }}[\mathcal{C}]\right) \equiv \inf _{w \in \mathcal{Z}_{N}}\left\|u^{\text {true }}[\mathcal{C}]-w\right\|$ is small and in particular decays rapidly with $N$. Towards this end, we consider a construction of $\mathcal{Z}_{N}$ based on the (prior) best knowledge as reflected in a parametrized best-knowledge model.

We first introduce a parameter $\mu \in \mathcal{D} \subset \mathbb{R}^{P}$; here $\mathcal{D}$ is the parameter domain associated with the anticipated uncertainty in the parameter of the best-knowledge model. We next introduce a parametrized form $G^{\mu}: \mathcal{U} \times \mathcal{U} \rightarrow \mathbb{C}$; we assume the form is antilinear in the second argument. We then define the best-knowledge solution $u^{\mathrm{bk}, \mu}$ as the solution of a parametrized PDE: given $\mu \in \mathcal{D}$, find $u^{\mathrm{bk}, \mu} \in \mathcal{U}$ such that

$$
G^{\mu}\left(u^{\mathrm{bk}, \mu}, v\right)=0 \quad \forall v \in \mathcal{U} ;
$$


we assume that the problem is well-posed in the sense that $u^{\mathrm{bk}, \mu}$ exists and is unique for any $\mu \in \mathcal{D}$. The parametrized PDE induces the parametric manifold

$$
\mathcal{M}^{\mathrm{bk}} \equiv\left\{u^{\mathrm{bk}, \mu} \in \mathcal{U} \mid \mu \in \mathcal{D}\right\}
$$

Note that the manifold reflects the state uncertainty induced by the anticipated uncertainty in the parameter of the model. We define the model error as the best-fit-over-manifold error:

$$
\epsilon_{\bmod }^{\mathrm{bk}, \mathcal{D}}\left(u^{\text {true }}[\mathcal{C}]\right) \equiv \inf _{w \in \mathcal{M}^{\mathrm{bk}}}\left\|u^{\text {true }}[\mathcal{C}]-w\right\|=\inf _{\mu \in \mathcal{D}}\left\|u^{\text {true }}[\mathcal{C}]-u^{\mathrm{bk}, \mu}\right\| \equiv\left\|u^{\text {true }}[\mathcal{C}]-F_{\mathcal{M}^{\mathrm{bk}}} u^{\text {true }}[\mathcal{C}]\right\|
$$

where $F_{\mathcal{M}^{\mathrm{bk}}}: \mathcal{U} \rightarrow \mathcal{M}^{\mathrm{bk}}$ identifies the infimizer; here the superscript bk, $\mathcal{D}$ on $\epsilon_{\bmod }^{\mathrm{bk}, \mathcal{D}}\left(u^{\text {true }}[\mathcal{C}]\right)$ emphasizes that the model error is associated with the manifold $\mathcal{M}^{\mathrm{bk}}$ induced by the parameter domain $\mathcal{D}$. We would like to choose the parametrized form $G^{\mu}(\cdot, \cdot)$ and the parameter domain $\mathcal{D}$ to minimize the model error. However, we must also consider approximation of $\mathcal{M}^{\text {bk }}$.

Based on the (prior) best-knowledge encoded in $\mathcal{M}^{\text {bk }}$, we construct a sequence of linear spaces $\mathcal{Z}_{N}$, $N=1, \ldots, N_{\max }$. In this work we consider the WeakGreedy procedure described in [16]. In short, the procedure identifies snapshots on the manifold,

$$
\forall n=1, \ldots, N, \quad \hat{\mu}_{n} \in \mathcal{D} \rightarrow u^{\mathrm{bk}, \hat{\mu}_{n}} \equiv \zeta_{n}
$$

such that $\mathcal{Z}_{N} \equiv \operatorname{span}\left\{\zeta_{n}\right\}_{n=1}^{N}$ approximates well the elements in $\mathcal{M}^{\mathrm{bk}}$. More precisely, the algorithm ensures that the discretization error

$$
\epsilon_{\mathrm{disc}, N}^{\mathrm{bk}, \mathcal{D}} \equiv \sup _{w \in \mathcal{M}^{\mathrm{bk}}} \inf _{z \in \mathcal{Z}_{N}}\|w-z\|=\sup _{\mu \in \mathcal{D}} \inf _{z \in \mathcal{Z}_{N}}\left\|u^{\mathrm{bk}, \mu}-z\right\|
$$

decays rapidly with $N$ and comparable to the Kolmogorov $N$-width associated with $\mathcal{M}^{\text {bk }}$ [3]; here the superscript bk, $\mathcal{D}$ on $\epsilon_{\mathrm{disc}, N}^{\mathrm{bk}, \mathcal{D}}$ emphasizes that the discretization error is the maximum error over the parametric manifold $\mathcal{M}^{\mathrm{bk}}$ induced by the parameter domain $\mathcal{D}$. Note we prefer the linear space $\mathcal{Z}_{N}$ rather than the manifold $\mathcal{M}^{\text {bk }}$ for purposes of online computational efficiency.

We may now decompose the background best-fit error into two contributions:

$$
\begin{aligned}
\epsilon_{N}^{\text {bk }}\left(u^{\text {true }}[\mathcal{C}]\right) & =\inf _{w \in \mathcal{Z}_{N}}\left\|u^{\text {true }}[\mathcal{C}]-w\right\| \leq\left\|u^{\text {true }}[\mathcal{C}]-\Pi_{\mathcal{Z}_{N}} F_{\mathcal{M}^{\text {bk }}} u^{\text {true }}[\mathcal{C}]\right\| \\
& \leq\left\|u^{\text {true }}[\mathcal{C}]-F_{\mathcal{M}^{\text {bk }}} u^{\text {true }}[\mathcal{C}]\right\|+\left\|F_{\mathcal{M}^{\text {bk }}} u^{\text {true }}[\mathcal{C}]-\Pi_{\mathcal{Z}_{N}} F_{\mathcal{M}^{\text {bk }}} u^{\text {true }}[\mathcal{C}]\right\| \\
& \leq \epsilon_{\bmod }^{\text {bk }}\left(u^{\text {true }}[\mathcal{C}]\right)+\epsilon_{\text {disc }, N}^{\text {bk }, \mathcal{D}}
\end{aligned}
$$

The first term $\epsilon_{\text {mod }}^{\mathrm{bk} \mathcal{D}}\left(u^{\text {true }}[\mathcal{C}]\right)$ is the modeling error, which arises from the fact that we cannot anticipate all forms of the uncertainty present in the system and/or cannot represent the uncertainty parametrically; hence, in general, $u^{\text {true }}[\mathcal{C}] \notin \mathcal{M}^{\text {bk }}$ and $\epsilon_{\bmod }^{\text {bk } \mathcal{D}}\left(u^{\text {true }}[\mathcal{C}]\right) \neq 0$. In particular, the modeling error can be large if the system exhibits unanticipated and unmodeled dynamics. The second term is the discretization error, which arises from the approximation of the manifold $\mathcal{M}^{\text {bk }}$ by a finite dimensional linear space $\mathcal{Z}_{N}$; in general, $\mathcal{M}^{\text {bk }} \not \subset \mathcal{Z}_{N}$ and $\epsilon_{\mathrm{disc}, N}^{\mathrm{bk}, \mathcal{D}} \neq 0$ - however, the WeakGreedy procedure ensures that the discretization error decays rapidly with $N$, in any event at a rate commensurate with the Kolmogorov $N$-width as proven in [3, 4,8].

The particular procedure discussed above is one of many procedures for the construction of the background space; we refer to [16] for alternatives.

Remark 5. The form of the model error $\epsilon_{\bmod }^{b k, \mathcal{D}}\left(u^{\text {true }}[\mathcal{C}]\right)$ and the discretization error $\epsilon_{\mathrm{disc}, N}^{b k, \mathcal{D}}$ in the decomposition (16) guides the selection of the parametrized best-knowledge model and the discretization procedure, respectively. This error decomposition, however, does not necessarily provide a sharp characterization of the background best-fit error. In particular, an alternate decomposition based on the modified definition of the model error as $\inf _{w \in \operatorname{span}\left\{\mathcal{M}^{k k}\right\}}\left\|u^{\text {true }}[\mathcal{C}]-w\right\|$ - where the best-fit is sought over the span of the manifold $\mathcal{M}^{b k}$ - provides a sharper characterization. However, this later form, while convenient for error analysis, 
is not particularly intuitive and does not guide the selection of the parametrized best-knowledge model or the discretization procedure.

We also introduce the prior prediction associated with our best knowledge of the problem in the absence of data. We in particular define our prior prediction as the parametrized best-knowledge model instantiated for the best-knowledge parameter $\mu^{\mathrm{bk}}: u^{\mathrm{bk}, \mu^{\mathrm{bk}}}$. We then define the error in the prior prediction as $\epsilon_{\text {mod }}^{\mathrm{bk},{ }^{\mathrm{bk}}} \equiv\left\|u^{\mathrm{true}}[\mathcal{C}]-u^{\mathrm{bk}, \mu^{\mathrm{bk}}}\right\|$. We emphasize that this prior prediction error - associated with $u^{\mathrm{bk}, \mu^{\mathrm{bk}}}\left(u^{\mathrm{true}}[\mathcal{C}]\right)$ corresponding to the specific parameter $\mu^{\mathrm{bk}}$ — is different from, and is no less than, the best-fit-over-manifold error $\epsilon_{\bmod }^{\text {bk }, \mathcal{D}}\left(u^{\text {true }}[\mathcal{C}]\right) \equiv \inf _{\mu \in \mathcal{D}}\left\|u^{\text {true }}[\mathcal{C}]-u^{\text {bk }, \mu}\right\|$ - associated with the parametric manifold $\mathcal{M}^{\text {bk }}$ induced by the parameter domain $\mathcal{D}$. We elaborate the distinction using the superscripts.

\subsection{Update Spaces}

As discussed in Section 3.4, the update space $\mathcal{U}_{M}$ must provide $\left(\mathrm{R}_{\mathcal{U}_{M}} 1\right)$ primary stability, $\left(\mathrm{R}_{\mathcal{U}_{M}} 2\right)$ secondary approximation, and $\left(\mathrm{R}_{\mathcal{U}_{M}} 3\right)$ well-conditioned observations. Towards this end, we employ the stabilitymaximizing greedy algorithm SGreedy introduced in [16]. In short, from a library $\mathcal{L}$ of candidate observation functionals, the algorithm sequentially chooses the observation functionals to maximize the stability constant $\beta_{N, M}$ for each $M$. In particular, for localized observations considered in this work, the library $\mathcal{L}$ consists of functionals associated with different observation centers, and the SGreedy algorithm selects a sequence of observation centers that maximizes the stability constant $\beta_{N, M}$.

The SGreedy algorithm explicitly addresses the requirement on primary stability, $\left(\mathrm{R}_{\mathcal{U}_{M}} 1\right)$. The SGreedy algorithm also addresses implicitly the requirement on well-conditioned observations, $\left(\mathrm{R}_{\mathcal{U}_{M}} 3\right)$; the maximization of the stability constant results in the selection of observation functionals that are distinct from each other, which constitutes well-conditioned observations. The SGreedy algorithm does not address $\left(\mathrm{R}_{\mathcal{U}_{M}} 2\right)$; however, we find that the convergence with the number of observations $M$ is slow in any event, and hence we focus on providing stability.

Again, the particular procedure discussed above is one of many procedures for the construction of the update space; we refer to [16] for alternatives, including the SGreedy' algorithm designed to balance the criteria on primal stability $\left(\mathrm{R}_{\mathcal{U}_{M}} 1\right)$ and secondary approximation $\left(\mathrm{R}_{\mathcal{U}_{M}} 2\right)$.

\section{Applichtion: Raised-Box Acoustic Resonator}

\subsection{Physical System}

We consider the application of the PBDW framework to a physical system: a raised-box acoustic resonator. The system is depicted in Figure 2(a). We note that this problem was first considered in [16]; a detailed description of the physical system, data-acquisition procedure, and best-knowledge mathematical model is provided in [16]. We provide here only a brief overview of the raised-box acoustic resonator system and the experimental protocol. We instead focus on providing a more detailed analysis and interpretation of the data assimilation results.

\subsection{System Configuration}

By way of preliminaries, we introduce a nondimensional frequency,

$$
\tilde{k} \equiv \frac{2 \pi \tilde{f}^{\operatorname{dim}} \tilde{r}_{\mathrm{spk}}^{\operatorname{dim}}}{\tilde{c}_{0}^{\operatorname{dim}}}
$$

where $\tilde{f}^{\mathrm{dim}}$ is the driving frequency of the speaker, $\tilde{r}_{\mathrm{spk}}^{\mathrm{dim}} \approx 2.54 \mathrm{~cm}$ is the radius of the speaker diaphragm, and $\tilde{c}_{0}^{\operatorname{dim}} \approx 346 \mathrm{~m} / \mathrm{s}$ is the speed of sound. Here the tilde $(\tilde{)})$ implies that the quantity is a measured quantity. Note that $\tilde{k}$ may also be interpreted as the measured nondimensional wavenumber.

We then define a system configuration

$$
\begin{aligned}
\mathcal{C}= & (\tilde{k}, \text { box dimensions, box material properties, speaker placement }, \\
& \text { speaker characteristics, temperature, extra-box environment, } \ldots) .
\end{aligned}
$$




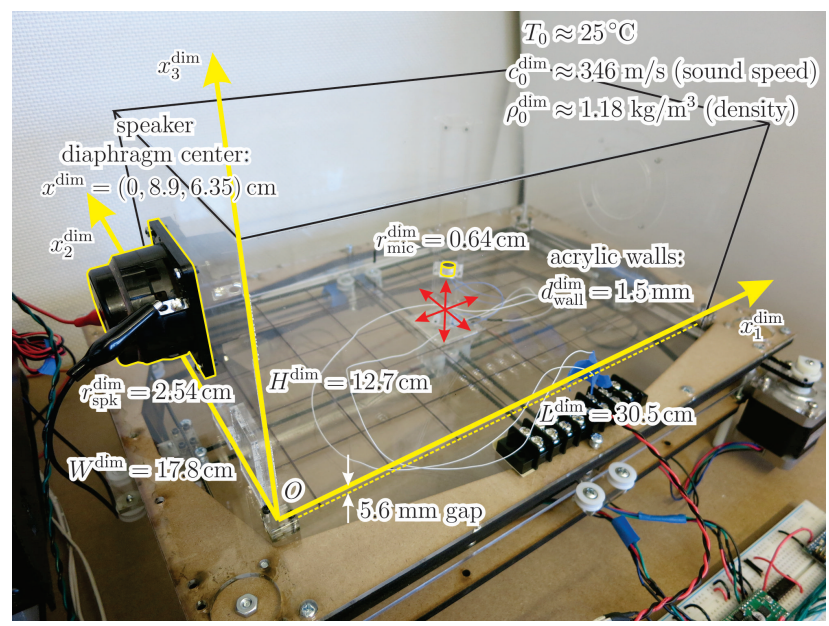

(a) physical system

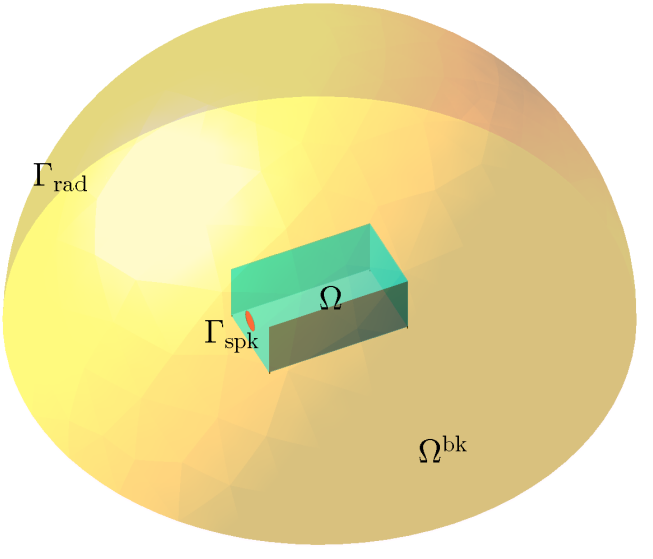

(b) computational domain

FiguRE 2. The raised-box acoustic resonator and the robotic observation platform: (a) the physical model, and (b) the best-knowledge computational domain. The figures reproduced with permission from [16].

We distinguish the nondimensional frequency $\tilde{k}$ - which we will actively vary and control — from all other properties that define the configuration and over which we have no control. To make the distinction more explicit, we denote the configuration of the acoustic resonator driven at the nondimensional frequency $\tilde{k}$ by $\mathcal{C}_{\tilde{k}}$.

\subsection{Robotic Observation Platform}

We perform autonomous, rapid, and accurate data acquisition using a robotic observation platform. A microphone mounted on a three-axis actuation platform measures the time-harmonic acoustic pressure of the system in the configuration $\mathcal{C}_{\tilde{k}}$ at the specified location. We next convert the time-harmonic signal to complex pressure, $\tilde{p}_{m}^{\mathrm{dim}}$, through a least-squares fit. The error in the time-harmonic complex pressure is estimated to be about 5\%. This error includes not only the noise-induced stochastic error considered in Section 3.3, but also the error associated with the calibration of the instruments. The error also includes the effect of inevitable variations in the configuration during the data-acquisition sequence; in actual physical experiments, unlike in the ideal abstract setting we have considered, we cannot perfectly maintain the configuration $\mathcal{C}_{\tilde{k}}$ invariant. We refer to [16] for detailed descriptions of the microphone calibration and validation procedures. We take these pressure observations as effectively "exact"; more precisely, we will only attempt to assess the accuracy of the data assimilation procedure to the $5 \%$ error level consistent with the observations.

We now normalize $\tilde{p}_{m}^{\operatorname{dim}}\left[\mathcal{C}_{\tilde{k}}\right]$ as

$$
\tilde{y}_{m}\left[\mathcal{C}_{\tilde{k}}\right]=\frac{\tilde{p}_{m}^{\operatorname{dim}}\left[\mathcal{C}_{\tilde{k}}\right]}{\tilde{\rho}_{0}^{\operatorname{dim}} \tilde{\mathcal{C}}_{0}^{\operatorname{dim}} V_{\mathrm{spk}}^{\mathrm{bk} \operatorname{dim}}(\tilde{k})},
$$

where, $\tilde{\rho}_{0}^{\operatorname{dim}} \approx 1.18 \mathrm{~kg} / \mathrm{m}^{3}$ is the density of air, and $V_{\mathrm{spk}}^{\mathrm{bk}, \operatorname{dim}}(\tilde{k})$ is the speaker diaphragm velocity deduced from the speaker best-knowledge model introduced below. For the purpose of PBDW estimation and subsequent assessment, we collect 84 observations on a $7 \times 4 \times 3$ axis-aligned grid for each nondimensional frequency $\tilde{k} \in[0.3,0.7]$.

\subsection{Best-Knowledge Model and Imperfections}

We now introduce our parametrized best-knowledge model. The model is defined over an extended domain $\Omega^{\mathrm{bk}} \subset \Omega$ shown in Figure 2(b), such that a radiation boundary condition can be specified away from the 
acoustic resonator itself; the details of this "super-domain" formulation is provided in [16]. The model consists of two parameters: the nondimensional frequency $k \in \mathcal{D}_{k} \equiv[0.3,0.7]$; the speaker amplification and phase-shift factor $\gamma \in \mathcal{D}_{\gamma} \equiv \mathbb{C}$. We now introduce the best-knowledge nondimensionalized pressure field,

$$
u^{\mathrm{bk}, \mu} \equiv \frac{p^{\mathrm{bk}, \mu, \operatorname{dim}}}{\rho_{0}^{\operatorname{dim}} c_{0}^{\operatorname{dim}} V_{\mathrm{spk}}^{\mathrm{bk}, \operatorname{dim}}(k)},
$$

as the solution of the following weak statement: given $\mu \equiv(k, \gamma) \in \mathcal{D}_{k} \times \mathcal{D}_{\gamma} \equiv \mathcal{D}$, find $u^{\mathrm{bk}, \mu} \in \mathcal{U}^{\mathrm{bk}} \equiv H^{1}\left(\Omega^{\mathrm{bk}}\right)$ such that

$$
a\left(u^{\mathrm{bk}, \mu}, v ; \mu\right)=f(v ; \mu) \quad \forall v \in \mathcal{U}^{\mathrm{bk}},
$$

where

$$
\begin{aligned}
a(w, v ; \mu) & \equiv \int_{\Omega} \nabla w \cdot \nabla \bar{v} d x-k^{2} \int_{\Omega} w \bar{v} d x+\left(i k+\frac{1}{R}\right) \int_{\Gamma_{\mathrm{rad}}} w \bar{v} d s \quad \forall w, v \in \mathcal{U}^{\mathrm{bk}}, \\
f(v ; \mu) & \equiv i k \gamma \int_{\Gamma_{\mathrm{spk}}} 1 \bar{v} d s \quad \forall v \in \mathcal{U}^{\mathrm{bk}} .
\end{aligned}
$$

We model the time-harmonic propagation of the sound wave in air by the Helmholtz equation. We model the walls of the raised-box resonator by a homogeneous Neumann boundary condition, which imposes infinite acoustic impedance. We model the time-harmonic forcing generated by the speaker as a uniform Neumann condition over $\Gamma_{\mathrm{spk}}$ of magnitude $k \rightarrow \gamma V^{\mathrm{bk}, \operatorname{dim}}(k)$, where $V^{\mathrm{bk}, \operatorname{dim}}(k)$ is derived from a second-order oscillator model for the electromechanical speaker; note this model appears in our equations indirectly through the nondimensionalization of (18) and directly in the Neumann condition on $\Gamma_{\mathrm{spk}}$ through the $\gamma$ (amplitude) and "1" (spatial uniformity). We model the radiation into free space by a first-order radiation condition on $\Gamma_{\text {rad }}$; the radiation term also ensures that the problem is well-posed for all $\mu \in \mathcal{D}$. We approximate the solution by a finite element solution associated with a 35,325-element $\mathbb{P}^{3}$ space.

While we choose the best-knowledge model to reflect our best-knowledge of the physical problem (subject to experimental and computational constraints), the model is not perfect. Here we identify some anticipated model imperfections and hence unmodeled physics.

The first set of imperfections is associated with our speaker model, $k \rightarrow \gamma V^{\text {bk,dim }}(k)$. First, the real speaker may exhibit non-rigid diaphragm motion, which is not captured by our uniform-velocity speaker model. Second, the real speaker may exhibit nonlinear response, which is not captured by our secondorder harmonic oscillator model. Third, the real speaker may experience feedback from the variation in the pressure inside the box, which is not captured by our Neumann speaker model. Fourth, the real speaker may not be mounted perfectly symmetrically about the $x_{2}$ plane, unlike the perfectly centered $\Gamma_{\mathrm{spk}}$ of our mathematical model. As we will see shortly, this unmodeled asymmetry in the speaker can have a significant consequence: on one hand, every solution to our mathematical model is symmetric about the $x_{2}$ plane, and hence our background space is symmetric about the $x_{2}$ plane; on the other hand, the slightest asymmetry in the speaker location can excite non-symmetric modes, especially when the speaker is operating near an anti-symmetric resonance frequency.

The second set of imperfections is associated with our wall model. First, the real wall is elastic and has a finite and spatially varying acoustic impedance, which is not captured by the perfectly rigid (infinite impedance) wall of our best-knowledge model. Second, the real elastic wall provides a dissipative mechanism through damping which is again not captured by the rigid wall of our best-knowledge model. Third, the real raised-box enclosure has fasteners and joints not captured by the homogeneous wall of our best-knowledge model.

The third set of imperfections is associated with our radiation model. The real acoustic resonator is not placed in a free space, and the pressure field inside the box can be affected by various environmental factors.

We now turn to the prior prediction and prior prediction error. For the prior prediction, we take the best-knowledge solution $u^{\mathrm{bk}, \mu}$ at $\mu=\mu^{\mathrm{bk}}=\left(k^{\mathrm{bk}}=\tilde{k}, \gamma^{\mathrm{bk}}=1\right)$. The prior prediction shall perforce suffer from the same imperfections as the parametrized best-knowledge model. However, in addition, errors will be 


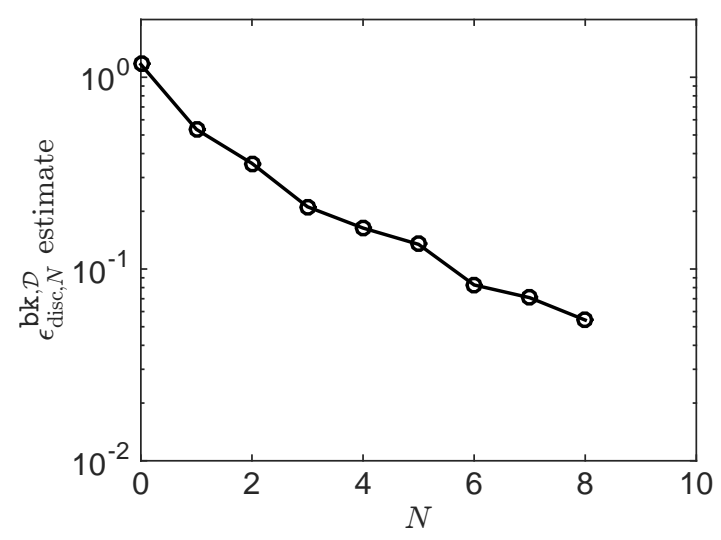

Figure 3. Convergence of the discretization error.

incurred due to the choice of $\mu^{\mathrm{bk}}$. (It is less that the prior prediction suffers from an incorrect choice of these parameters, and more that the best-fit-over-manifold can exploit flexibility in these parameters within the Helmholtz structure. In particular, the best-fit-over-manifold can benefit from a choice of $k$ different from $k^{\mathrm{bk}}=\tilde{k}$ (even though $\tilde{k}$ is an accurate measurement of the actual frequency) and a choice of $\gamma$ different from $\gamma^{b k}=1$ as a way to represent a shift in resonance frequency and modification of amplitude and phase due in fact to the imperfect modeling of wall interactions, damping, and speaker.)

\subsection{PBDW Formulation}

We first introduce Hilbert space $\mathcal{U} \equiv H^{1}(\Omega)$ endowed with a weighted $H^{1}$ inner product

$$
(w, v) \equiv \int_{\Omega} \nabla w \cdot \nabla \bar{v} d x+k_{\mathrm{ref}}^{2} \int_{\Omega} w \bar{v} d x \quad \forall w, v \in \mathcal{U},
$$

and the associated induced norm $\|w\| \equiv \sqrt{(w, w)}$; we choose $k_{\text {ref }}=0.5$ as the reference frequency.

We next introduce the background space. The background space is generated in two steps: we first apply the WeakGreedy algorithm to $\mathcal{M}^{\mathrm{bk}} \equiv\left\{u^{\mathrm{bk}, \mu} \mid \mu \in \mathcal{D}\right\}$ to obtain hierarchical reduced basis spaces $\mathcal{Z}_{N}^{\mathrm{bk}} \equiv \operatorname{span}\left\{u^{\mathrm{bk}, \hat{\mu}_{n}}\right\}_{n=1}^{N}, N=1, \ldots, 8 \equiv N_{\max }$; we then restrict the functions over $\Omega^{\mathrm{bk}}$ in $\mathcal{Z}_{N}^{\mathrm{bk}}$ to the domain of interest $\Omega$ to form $\mathcal{Z}_{N} \equiv\left\{z \in \mathcal{U}\left|z=z^{\mathrm{bk}}\right|_{\Omega}, z^{\mathrm{bk}} \in \mathcal{Z}_{N}^{\mathrm{bk}}\right\}, N=1, \ldots, N_{\max }$. Note that this procedure, which first computes the basis over $\Omega^{\mathrm{bk}}$ and then restricts the basis to $\Omega \subset \Omega^{\mathrm{bk}}$, can generate an ill-conditioned basis if any two basis functions differ only over $\Omega^{\text {bk }} \backslash \Omega$; a more stable approach is to work on functions restricted to $\Omega \subset \Omega^{\mathrm{bk}}$ in the initial selection of the basis functions. However, this latter procedure requires non-trivial localization of residual and does not permit the direct application of the WeakGreedy algorithm, which operates on the PDE defined over $\Omega^{\mathrm{bk}}$. Note also that if the localized basis is ill-conditioned, then we could apply POD and discard unnecessary elements to improve the conditioning. In any event, we do not encounter this ill-conditioning issue in our raised-box acoustic resonator.

We show in Figure 3 the convergence of an estimate of the discretization error $\epsilon_{\mathrm{disc}, N}^{\mathrm{bk}, \mathcal{D}} \equiv \sup _{w \in \mathcal{M}^{\mathrm{bk}}} \inf _{z \in \mathcal{Z}_{N}} \| w-$ $z \|$; recall that $\left(\mathrm{R}_{\mathcal{Z}_{N}} 1\right)$, in view of (16), requires a small discretization error. Thanks to the judicious choice of the snapshots by the WeakGreedy algorithm, the discretization error decays rapidly with $N$.

We next introduce the observation functionals and the associated experimentally observable update space. We model the experimental observations provided by the microphone with Gaussians, $\ell_{m}^{\circ} \equiv \operatorname{Gauss}\left(\cdot ; x_{m}^{\mathrm{c}}, r_{m}=\right.$ 0.2 ). We then form a library $\mathcal{L}$ of 84 observation functionals consistent with the dataset described in Section 5.3. We apply the SGreedy algorithm to the library $\mathcal{L}$ and $\mathcal{Z}_{N_{\max }}$ to choose a sequence of observation functionals, $\ell_{m}^{\mathrm{o}}, m=1, \ldots, 48 \equiv M_{\max }$, that maximizes the stability constant $\beta_{N, M}$. We finally form the associated experimentally observable update spaces, $\mathcal{U}_{M} \equiv\left\{q_{m} \equiv R_{\mathcal{U}} \ell_{m}^{\circ}\right\}_{m=1}^{M}, M=1, \ldots, M_{\max }$. Note that the precise choice of the filter width $r_{m}$ is not important here since the microphone dimension is quite small compared to the wavelengths of interest. 


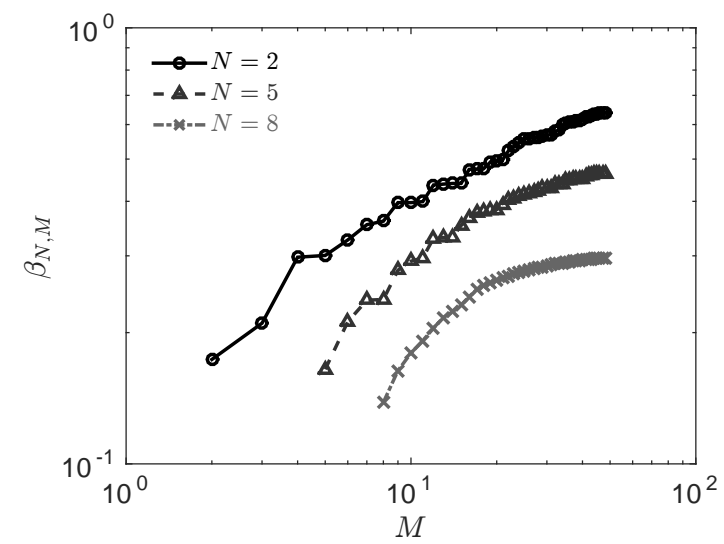

(a) inf-sup constant

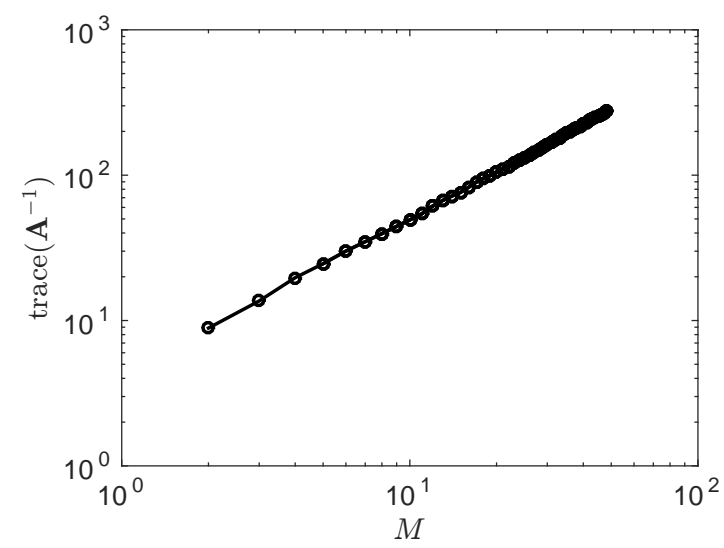

(b) observation-conditioning metric

Figure 4. Behavior of the stability constant $\beta_{N, M}$ and the observation-conditioning metric $\operatorname{trace}\left(\mathbf{A}^{-1}\right)$.

We show in Figure 4(a) the behavior of the stability constant $\beta_{N, M}$; recall that $\left(\mathrm{R}_{\mathcal{U}_{M}} 1\right)$ requires the infsup constant to be away from zero and close to the unity. We note that the inf-sup constant associated with the observation sequence identified by SGreedy is considerably larger than the inf-sup constant associated with a random sequence of observations, as demonstrated in [16]. The SGreedy algorithm ensures that this requirement is satisfied. We show in Figure 4(b) the behavior of the observation-conditioning metric trace $\left(\mathbf{A}^{-1}\right)$; recall that $\left(\mathrm{R}_{\mathcal{U}_{M}} 3\right)$ requires that trace $\left(\mathbf{A}^{-1}\right)$ is not too large. Because the transducers have relatively narrow filter width, and the maximization of the stability constant by SGreedy tends to result in the selection of observation centers that are distant from each other, the observations are well-conditioned; in particular, we do not observe the exponential divergence observed for some cases in Figure 1. In conjunction with the relatively small observation error of $5 \%$ as reported in Section 5.3, we conclude that the stochastic error is relatively small, and in any event does not grow exponentially with $M$.

\subsection{Real-Time In Situ State Estimation}

We now report a typical data assimilation result. Figure 5(a) shows the imaginary component of the time-harmonic pressure field for $\tilde{k}=0.69$ estimated from the PBDW formulation for a $N=7$ dimensional background space and $M=12$ observations. The observation points are depicted by the orange dots. Although the 12 observations arguably provide only sparse coverage relative to the complexity of the field, we are able to recover a qualitatively convincing pressure field thanks to the information provided by the background space. (We will quantitatively confirm in Section 5.7 that the estimated field is indeed a good approximation of the true pressure field.)

The PBDW formulation provides a full state estimation, and hence we may compute other derived fields of engineering interest, such as the sound intensity field depicted in Figure 5(b). For a time harmonic field, the sound intensity is related to the pressure field by

$$
I_{\mathrm{avg}}=\Re\left(\frac{-i}{4 \pi \tilde{\rho}_{0}^{\operatorname{dim}} \tilde{f} \operatorname{dim}} p_{N, M}^{\operatorname{dim}} \nabla \bar{p}_{N, M}^{\operatorname{dim}}\right),
$$

where $\Re(\cdot)$ takes the real component of the complex number, and $p_{N, M}^{\mathrm{dim}}$ is the dimensional pressure. We may readily post-process the pressure field to obtain the sound intensity field. We observe in Figure 5(b) that the sound intensity is highest near the speaker. We note that the sound intensity exhibits a rather complicated structure even for this relatively simple acoustics problem.

We make a few remarks about the timing associated with data acquisition, data assimilation, and rendering. The robotic observation platform requires on average little over 3 seconds per observation to reposition the microphone and to acquire the time harmonic signal. The acquisition of the $M=12$ observations hence 


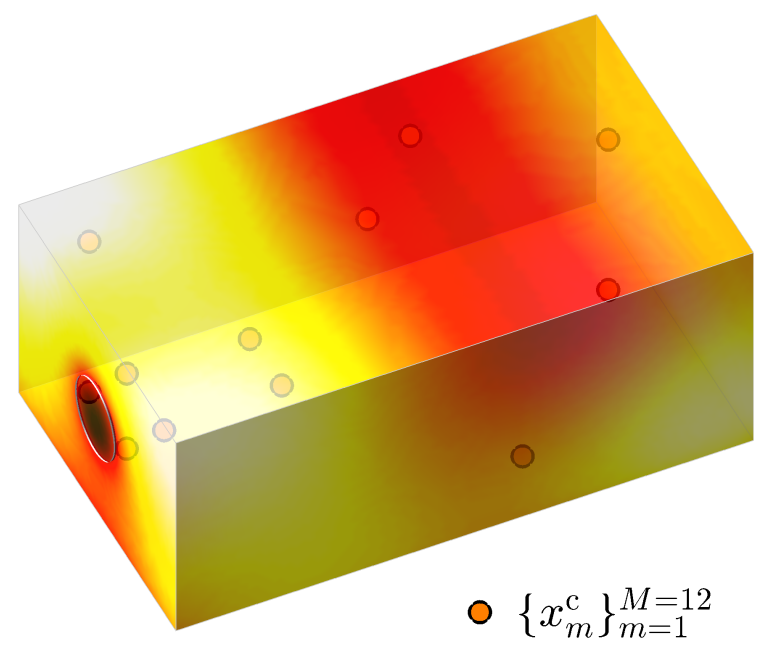

(a) pressure field

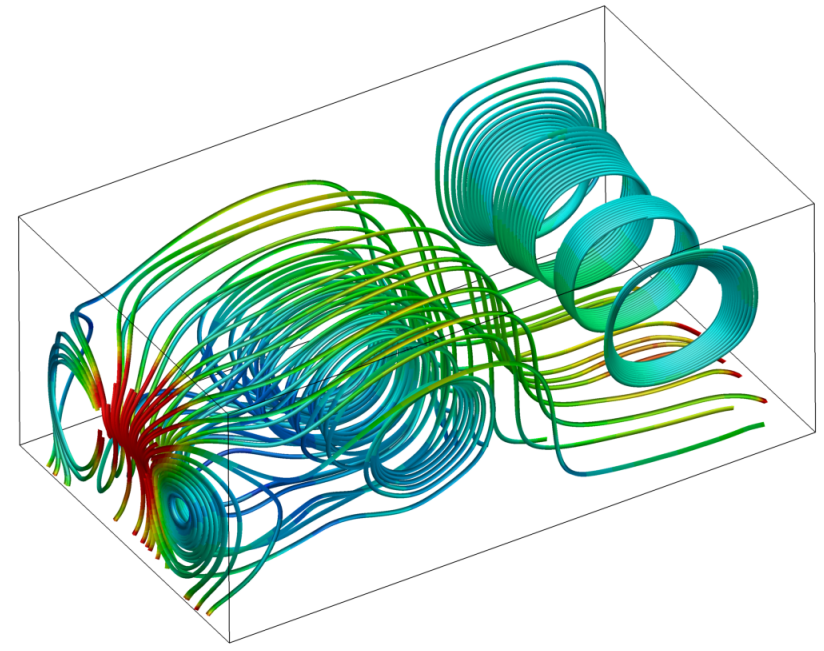

(b) intensity field

Figure 5. The $N=7, M=12$ PBDW estimate of the (a) pressure field and (b) intensity field for the normalized frequency of $\tilde{k}=0.69$.

takes approximately 40 seconds. The solution of the saddle system of size $N+M=19$ takes less than 0.1 milliseconds on a laptop. The rendering of the solution using the current implementation takes approximately 0.8 seconds; we speculate that this time can be significantly reduced by optimizing the rendering procedure at the software and hardware level. In any event, the total online time is dictated by the data acquisition step and is approximately $3 M$ seconds.

\subsection{Error Analysis and Interpretations}

\subsubsection{Assessment Procedure}

From the 84 observations we collect, we choose $J=36$ observations associated with observation functional centers $\left\{\xi_{m}^{c}\right\}_{j=1}^{J=36}$ as assessment observations. We ensure that the observation centers $\left\{x_{m}^{c}\right\}_{m=1}^{M_{\max }=48}$ used in the PBDW state estimation and the observation centers $\left\{\xi_{m}^{\mathrm{c}}\right\}_{j=1}^{J=36}$ used in the assessment are mutually exclusive: $\xi_{j}^{\mathrm{c}} \notin\left\{x_{m}^{\mathrm{c}}\right\}_{m=1}^{M_{\max }}, j=1, \ldots, J$. We then introduce the following pressure estimates associated with the assessment observations:

$$
\begin{aligned}
& P^{\text {prior }}(j ; \tilde{k}) \equiv \operatorname{Gauss}\left(u^{\mathrm{bk}, \mu^{\mathrm{bk}}=\left(k^{\mathrm{bk}} \equiv \tilde{k}, \gamma^{\mathrm{bk}} \equiv 1\right)} ; \xi_{j}^{\mathrm{c}}, 0.2\right), \\
& P_{N, M}^{*}(j ; \tilde{k}) \equiv \operatorname{Gauss}\left(u_{N, M}^{*}\left[\mathcal{C}_{\tilde{k}}\right] ; \xi_{j}^{\mathrm{c}}, 0.2\right), \\
& P^{\text {true }}(j ; \tilde{k})=(\text { nondimensionalized experimental observation }(17) \\
&\left.\quad \text { at } \xi_{j}^{\mathrm{c}} \text { for configuration } \mathcal{C}_{\tilde{k}}\right),
\end{aligned}
$$

where $\mathcal{C}_{\tilde{k}}$ specifies the experimental configuration. Throughout the assessment process, we take the assessment observation as the "truth"; more precisely, as noted in Section 5.3, we will only attempt to assess the state error to the observation error level, $\approx 5 \%$, such that the observations can serve as a surrogate for the truth. We finally introduce an a posteriori error indicator,

$$
E_{\mathrm{avg}}\left[\mathcal{C}_{\tilde{k}}\right] \equiv \sqrt{\frac{1}{J} \sum_{j=1}^{J}\left|P^{\operatorname{true}}(j ; \tilde{k})-P_{N, M}^{*}(j ; \tilde{k})\right|^{2}}
$$



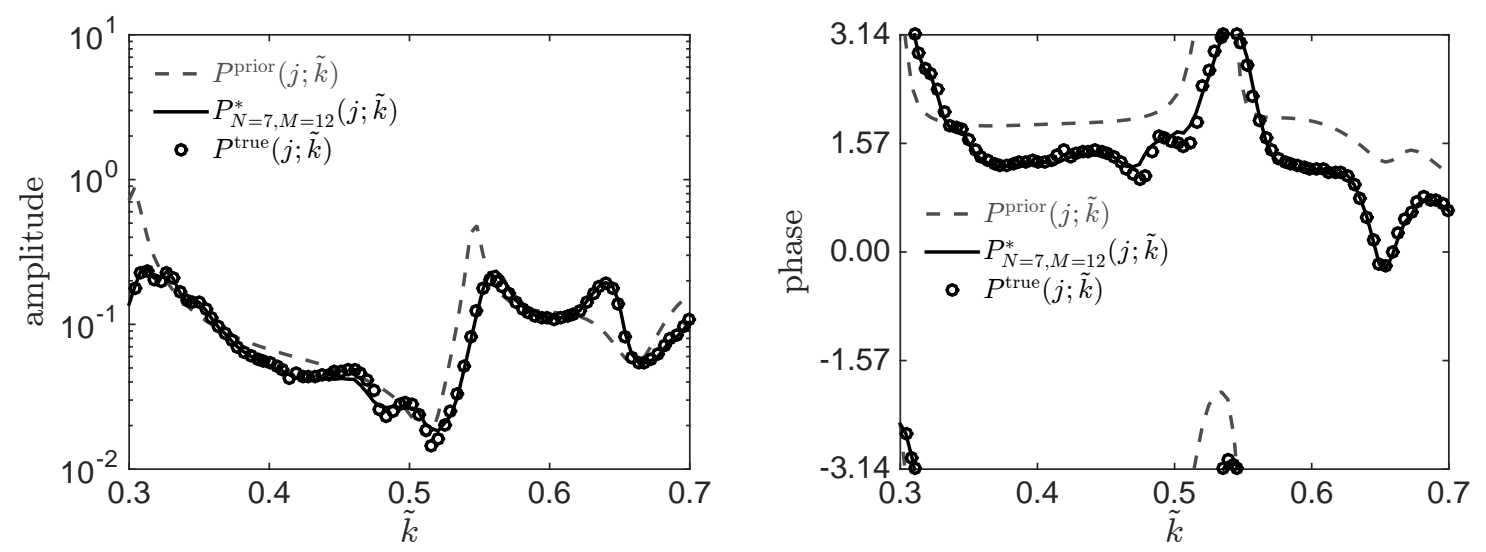

(a) $\xi_{j}^{\mathrm{c}}=(2.67,2.67,4.50)$
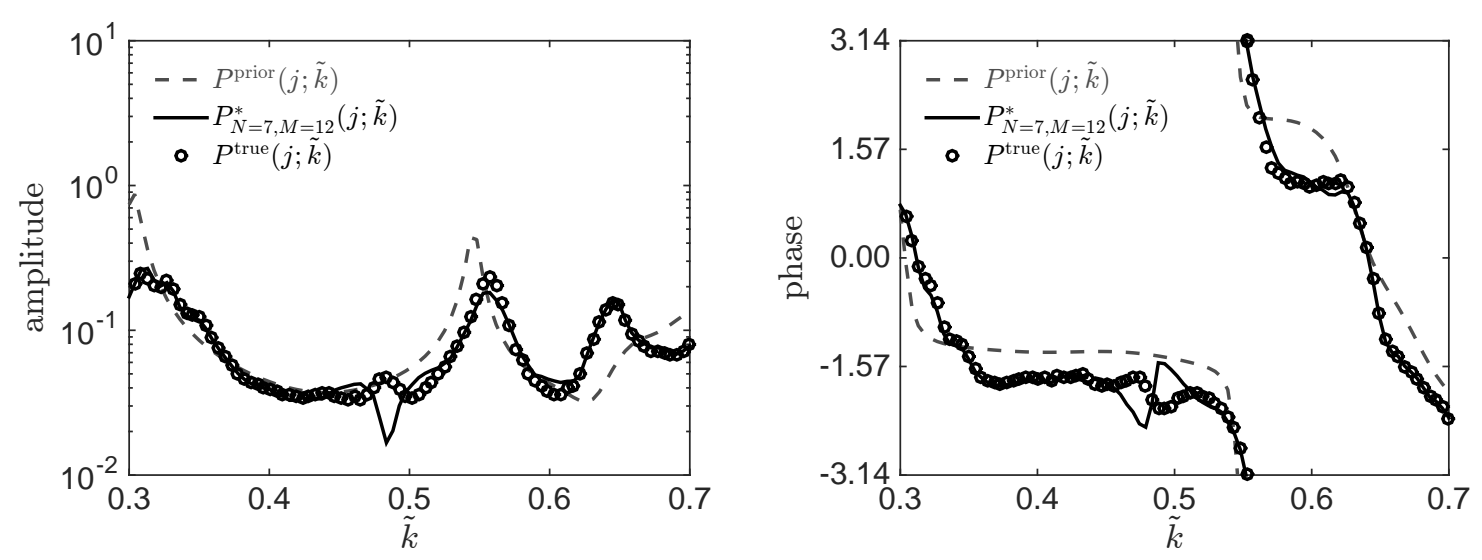

(b) $\xi_{j}^{\mathrm{c}}=(9.33,2.67,4.50)$

Figure 6. Frequency response (amplitude and phase) at the assessment center (a) $\xi_{j}^{\mathrm{c}}=$ $(2.67,2.67,4,50)$ and $(\mathrm{b}) \xi_{j}^{\mathrm{c}}=(9.33,2.67,4.50)$.

here, we recall that $N$ is the dimension of the background space, $M$ is the dimension of the observable space, $J$ is the number of assessment observations, and $\tilde{k}$ is the operating frequency. A formulation and analysis for this experimental a posteriori error estimate is provided in [19].

\subsubsection{Frequency Response}

We first study the frequency response obtained at a single observation point. In Figure 6(a), we compare three frequency response curves observed at $\xi_{j}^{\mathrm{c}}=(2.67,2.67,4.50)$ : the prior prediction; the prediction by the $N=7, M=12$ PBDW estimate; and the value observed by the microphone, which we take as the truth. We observe that the prior prediction differs considerably from the truth. The prior prediction overestimates the amplitude near the resonances and underestimates the resonance frequencies; we speculate the discrepancy arises from the use of the perfectly rigid wall in the best-knowledge model. The model also suffers from a phase shift. The PBDW estimate closely tracks the truth frequency response over the entire frequency range, in part due to the parametric flexibility provided in the speaker model, $\gamma \neq \gamma^{\mathrm{bk}}=1$, in part due to the intrinsic flexibility of model superposition provided by our linear space. (In actual practice, the former and latter are not distinguishable.)

We compare in Figure $6(\mathrm{~b})$ the frequency response curves observed at $\xi_{j}^{\mathrm{c}}=(9.33,2.67,4.50)$. We again observe that the prior prediction overestimates the resonance amplitude, underestimates resonance frequency, 


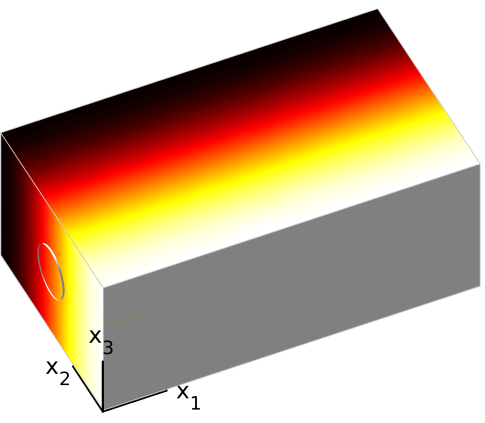

(a) $k^{\text {resonance }}=0.449$

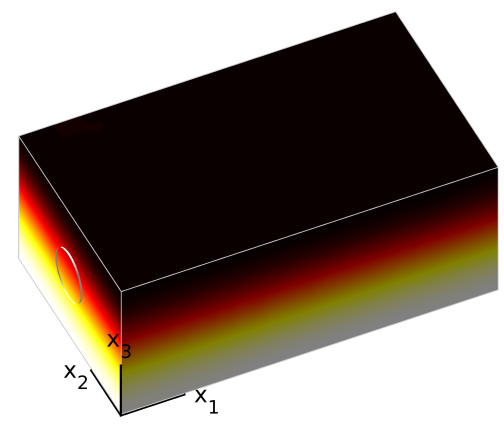

(d) $k^{\text {resonance }}=0.629$

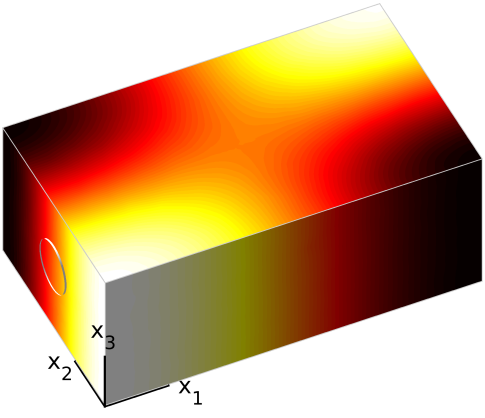

(b) $k^{\text {resonance }}=0.520$

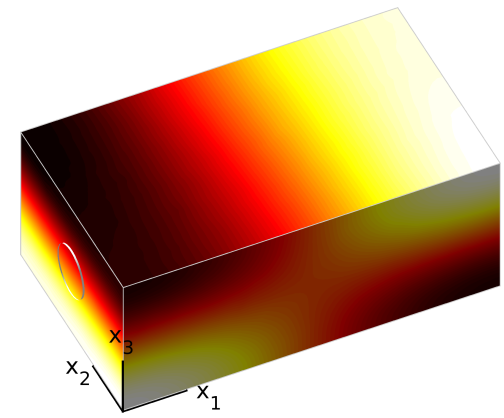

(e) $k^{\text {resonance }}=0.681$

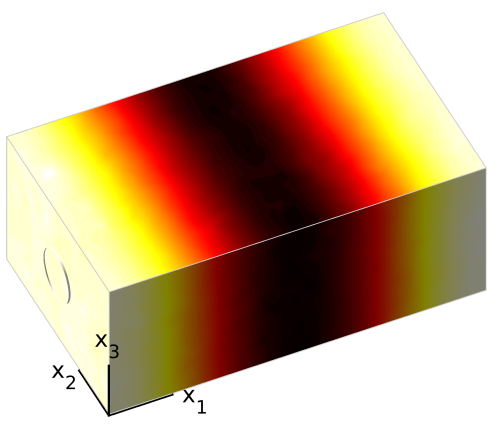

(c) $k^{\text {resonance }}=0.523$

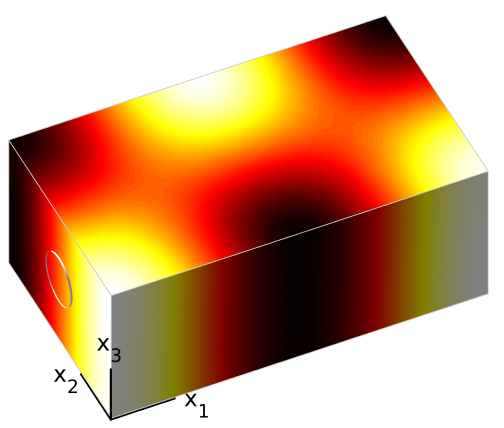

(f) $k^{\text {resonance }}=0.690$

FigurE 7. Resonance modes associated with the simple Neumann box.

and leads in the phase. The PBDW estimate again closely tracks the truth frequency response over the entire frequency range, except at near $\tilde{k}=0.48$.

We may explain the discrepancy observed near $\tilde{k}=0.48$ through the resonance modes associated with the acoustic resonator. To simplify the analysis, we consider here a simple box-only configuration with homogeneous Neumann boundary condition on all six sides of the box. The resonance modes in the frequency range $\mathcal{D}_{\tilde{k}} \equiv[0.3,0.7]$ are shown in Figure 7 . The inaccuracy of the PBDW estimate for $\tilde{k} \approx 0.48$ is due to the $x_{2}$-antisymmetric resonance mode shown in Figure $7(\mathrm{a})$. The physical system is inevitably asymmetric about the $x_{2}$-plane; for instance, the speaker is not mounted in the box precisely symmetrically and furthermore does not vibrate precisely symmetrically. Hence, in the physical system, $x_{2}$-antisymmetric resonance modes are excited. In contrast, the best-knowledge model, for all $\mu \in \mathcal{D}$, is perfectly symmetric about the $x_{2}$ plane, and hence only the symmetric resonance modes are excited. Hence, we expect significant (relative) model error for frequencies $\tilde{k}$ that are close to antisymmetric resonances but are far from symmetric resonances: $\tilde{k}=0.48$ satisfies this requirement.

\subsubsection{Convergence: $x_{2}$-Symmetric Resonance}

We now study the convergence behavior of the PBDW estimate for two different configurations: one near the $x_{2}$-symmetric resonance at $\tilde{k}=0.557$ and the other near the $x_{2}$-antisymmetric resonance at $\tilde{k}=0.479$. We first study the $x_{2}$-symmetric resonance at $\tilde{k}=0.557$. For this configuration, the $x_{2}$-asymmetry in the physical system is unimportant because $\tilde{k}$ is near the (shifted) symmetric resonance depicted in Figure 7 (b). (The shift in the resonance frequency arises due to the difference in the wall boundary conditions: the wall of the mathematical model is perfectly rigid; the real wall is elastic.) We hence expect $u^{\text {true }}\left[\mathcal{C}_{\tilde{k}}\right]$ to be close to the best-knowledge manifold $\mathcal{M}^{\text {bk }}$, and the model error $\epsilon_{\bmod }^{\text {bk, } \mathcal{D}}\left(u^{\text {true }}\left[\mathcal{C}_{\tilde{k}}\right]\right)$ to be small.

We show in Figure 8(a) the convergence of the PBDW estimate with the background space dimension $N$ and the number of observations $M$. We also show the errors associated with three different estimates: the error associated with the zero estimate $u_{N=0, M=0}^{*}=0$ (note that this "error" is in fact just the magnitude of $\left.u^{\text {true }}\left[\mathcal{C}_{\tilde{k}}\right]\right)$; the error associated with the prior prediction $u^{\mathrm{bk}, \mu^{\mathrm{bk}}}, \epsilon_{\bmod }^{\mathrm{bk}, \mu^{\mathrm{bk}}}\left(u^{\text {true }}\left[\mathcal{C}_{\tilde{k}}\right]\right)$; the error associated with 


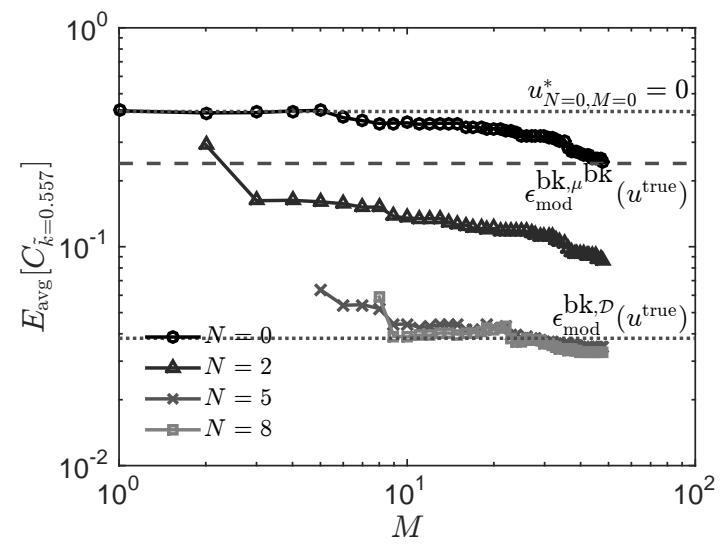

(a) convergence

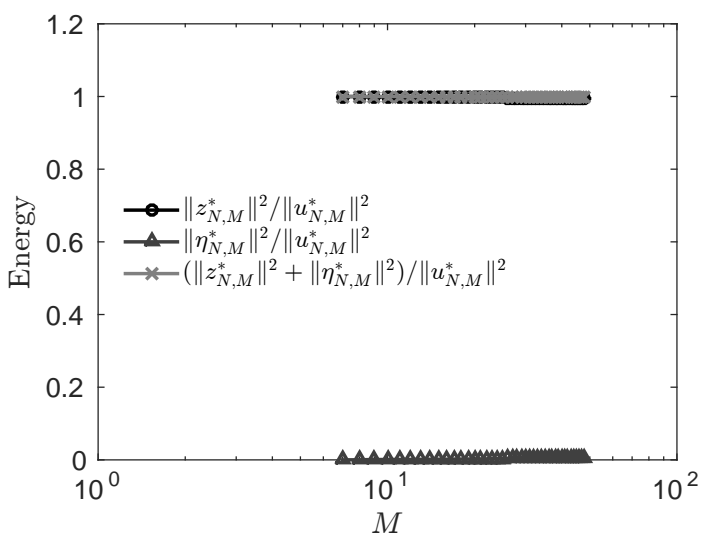

(b) energy fraction $(N=7)$

FiguRE 8. The convergence plot and the energy fraction decomposition for the $x_{2^{-}}$ symmetric resonance at $\tilde{k}=0.557$.

the best-fit-over-manifold estimate, $\epsilon_{\bmod }^{\mathrm{bk}, \mathcal{D}}\left(u^{\operatorname{true}}\left[\mathcal{C}_{\tilde{k}}\right]\right)$. Here, and in subsequent similar figures, $\epsilon_{\bmod }^{\mathrm{bk}, \mu^{\mathrm{bk}}}\left(u^{\mathrm{true}}\left[\mathcal{C}_{\tilde{k}}\right]\right)$ and $\epsilon_{\bmod }^{\mathrm{bk}, \mathcal{D}}\left(u^{\text {true }}\left[\mathcal{C}_{\tilde{k}}\right]\right)$ are approximated by (19) but for $P_{N, M}^{*}$ replaced by $P^{\text {prior }}$ and $P^{\text {best-fit }}$, respectively, where $P^{\text {prior }}$ is as defined in Section 5.7.1 and $P^{\text {best-fit }}$ is obtained by the nonlinear least-squares fit of the parametrized best-knowledge model with respect to all 84 observations. We note that the ratio of the error associated with any given estimate to the error associated with the zero estimate $u_{N=0, M=0}^{*}=0$ is in fact the relative error in the estimate.

We observe that the PBDW estimation error decreases rapidly as $N$ increases for a fixed $M(\geq N)$. Here, because the model error $\epsilon_{\bmod }^{\mathrm{bk}, \mathcal{D}}\left(u^{\text {true }}\left[\mathcal{C}_{\tilde{k}}\right]\right)$ is small, and the discretization error $\epsilon_{\mathrm{disc}, N}^{\mathrm{bk}, \mathcal{D}}$ tends to zero rapidly with $N$ by the construction of the background space $\mathcal{Z}_{N}$, the estimation error $\left\|u^{\text {true }}\left[\mathcal{C}_{\tilde{k}}\right]-u_{N, M}^{*}\left[\mathcal{C}_{\tilde{k}}\right]\right\|$ approaches $\epsilon_{\text {mod }}^{\text {bk }, \mathcal{D}}\left(u^{\text {true }}\left[\mathcal{C}_{\tilde{k}}\right]\right)$, quite small, rapidly with $N$. Specifically, the relative model error is approximately $10 \%$. Note that the model error, $\epsilon_{\bmod }^{\mathrm{bk}, \mathcal{D}}\left(u^{\text {true }}\left[\mathcal{C}_{\tilde{k}}\right]\right)$, is small not only due to the Helmholtz equation, which well represents solution shapes, but also due to the parameter flexibility described in Section 5.4. In this case, the background space $\mathcal{Z}_{N}$ provides approximation, and the observable space $\mathcal{U}_{M}$ provides stability; in particular, the role of $\mathcal{U}_{M}$ as the "update space" is limited.

We study the contribution of the background component $z_{N, M}^{*}\left[\mathcal{C}_{\tilde{k}}\right] \in \mathcal{Z}_{N}$ and the update component $\eta_{N, M}^{*}\left[\mathcal{C}_{\tilde{k}}\right] \in \mathcal{U}_{M}$ to the estimated state $u_{N, M}^{*}\left[\mathcal{C}_{\tilde{k}}\right]$ by computing the energy associated with each component. The result is shown in Figure 8(b). We observe that essentially all the energy is in the background component $z_{N, M}^{*}\left[\mathcal{C}_{\tilde{k}}\right]$; the update component $\eta_{N, M}^{*}\left[\mathcal{C}_{\tilde{k}}\right]$ contains an insignificant fraction of energy.

We finally show in Figures 9(a) and 9(b) the background field and update field, respectively, associated with the $N=7, M=48$ PBDW estimate. The background field captures the symmetric resonance mode; the update field does not exhibit any identifiable structure.

\subsubsection{Convergence: $x_{2}$-Antisymmetric Resonance}

We now study the $x_{2}$-antisymmetric resonance at $\tilde{k}=0.479$. For this configuration, the $x_{2}$-asymmetry in the physical system is important because $\tilde{k}$ is near the (shifted) antisymmetric resonance depicted in Figure 7(a) and is far from the (shifted) symmetric resonance depicted in Figure 7(b). We hence expect that $u^{\text {true }}\left[\mathcal{C}_{\tilde{k}}\right]$ will not be close to the best-knowledge manifold $\mathcal{M}^{\text {bk }}$ and that the model error $\epsilon_{\bmod }^{\text {bk, } \mathcal{D}}\left(u^{\text {true }}\left[\mathcal{C}_{\tilde{k}}\right]\right)$ will not be small.

We show in Figure 10(a) the convergence of the PBDW estimate with the background space dimension $N$ and the number of observations $M$. In contrast to the $x_{2}$-symmetric resonance case, we observe that the primary approximation by the background space is insufficient: as $N$ increases, the discretization error $\epsilon_{\mathrm{disc}, N}^{\mathrm{bk}, \mathcal{D}}$ rapidly tends to zero, but the estimation error $\left\|u^{\text {true }}\left[\mathcal{C}_{\tilde{k}}\right]-u_{N, M}^{*}\left[\mathcal{C}_{\tilde{k}}\right]\right\| \rightarrow \epsilon_{\bmod }^{\mathrm{bk}, \mathcal{D}}\left(u^{\text {true }}\left[\mathcal{C}_{\tilde{k}}\right]\right) \neq 0$ because 


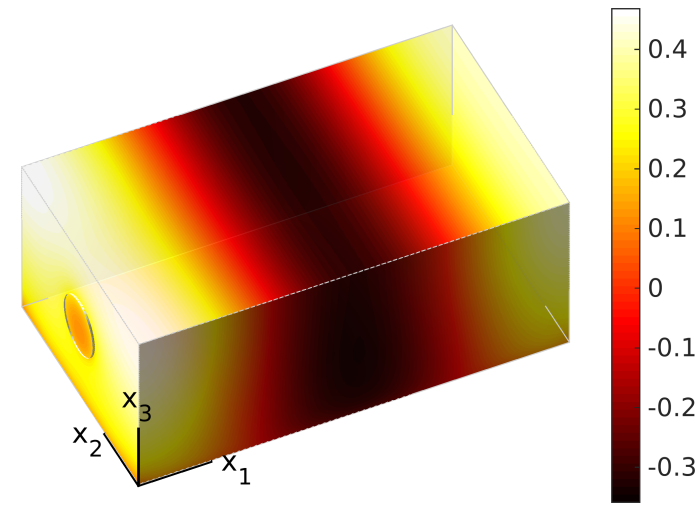

(a) $z_{N, M}^{*}\left[\mathcal{C}_{\tilde{k}}\right],\left\|z_{N, M}^{*}\right\|^{2}=46.13$

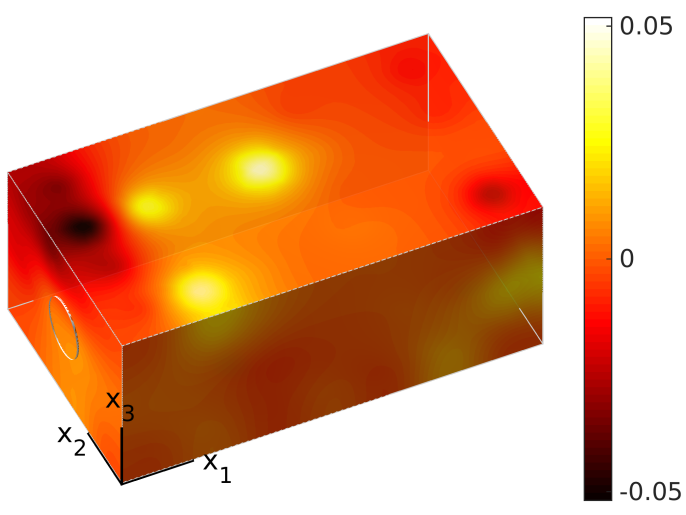

(b) $\eta_{N, M}^{*}\left[\mathcal{C}_{\tilde{k}}\right],\left\|\eta_{N, M}^{*}\right\|^{2}=0.29$

Figure 9. The (a) background and (b) update component of the $N=7, M=48$ PBDW estimate to the $x_{2}$-symmetric resonance configuration.

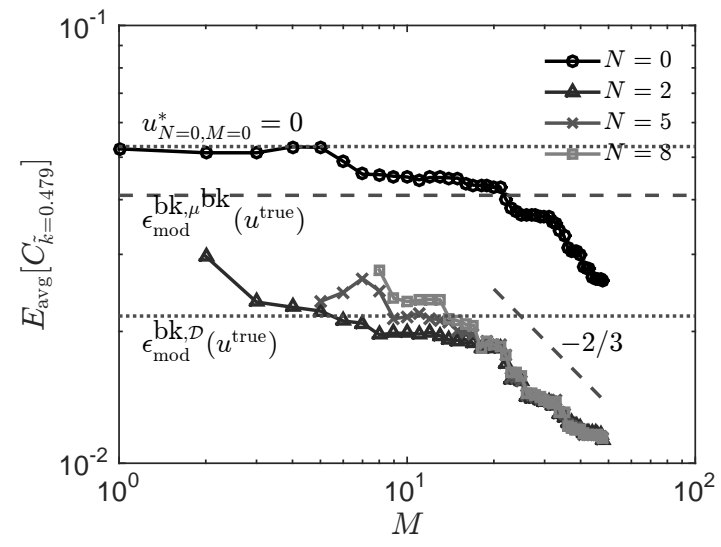

(a) convergence

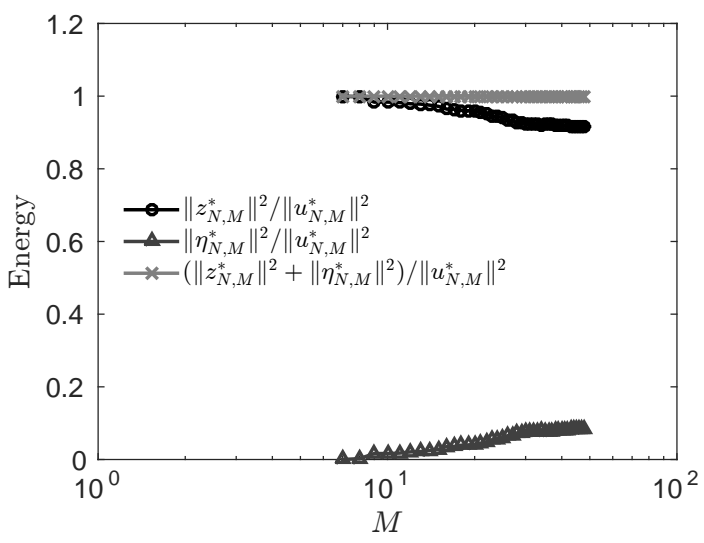

(b) energy fraction $(N=7)$

Figure 10. The convergence plot and the energy fraction decomposition for the $x_{2}$ antisymmetric resonance at $\tilde{k}=0.479$.

the model error is not small. Specifically, the relative model error is approximately $50 \%$. As a result, the convergence in $N$ stagnates for $N \geq 2$. We must thus rely on the secondary approximation by the update space $\mathcal{U}_{M}$ for convergence: as $M$ increases, we expect $\left\|u^{\text {true }}\left[\mathcal{C}_{\tilde{k}}\right]-u_{N, M}^{*}\left[\mathcal{C}_{\tilde{k}}\right]\right\|$ to decrease slowly. The space $\mathcal{U}_{M}$ provides approximation of the unmodeled physics. The observed convergence with $M$ for the $L^{2}(\Omega)$-related error indicator $E_{\text {avg }}\left[\mathcal{C}_{\tilde{k}=0.479}\right]$ is $M^{-2 / 3}=\left(M^{-1 / d}\right)^{2}$.

We show in Figure 10(b) the energy fraction of the background component $z_{N, M}^{*}\left[\mathcal{C}_{\tilde{k}}\right]$ and the update component $\eta_{N, M}^{*}\left[\mathcal{C}_{\tilde{k}}\right]$. Unlike the $x_{2}$-symmetric resonance case, in the $x_{2}$-antisymmetric resonance case there is non-negligible energy in the update component $\eta_{N, M}^{*}\left[\mathcal{C}_{\tilde{k}}\right]$ for a higher $M$. This is consistent with the fact that the update component captures the (here non-negligible) unmodeled physics.

We show in Figure 11(a) and 11(b) the background field and update field, respectively, associated with the $N=7, M=48 \mathrm{PBDW}$ estimate. Unlike in the $x_{2}$-symmetric resonance configuration, in the $x_{2^{-}}$ antisymmetric configuration the update field has a structure similar to the $x_{2}$-antisymmetric mode depicted in Figure 7 (a). We may conclude that the update field $\eta_{N, M}^{*}\left[\mathcal{C}_{\tilde{k}}\right]$ represents the $x_{2}$-antisymmetric resonance not included in the best-knowledge model (for any $\mu \in \mathcal{D}$ ). 


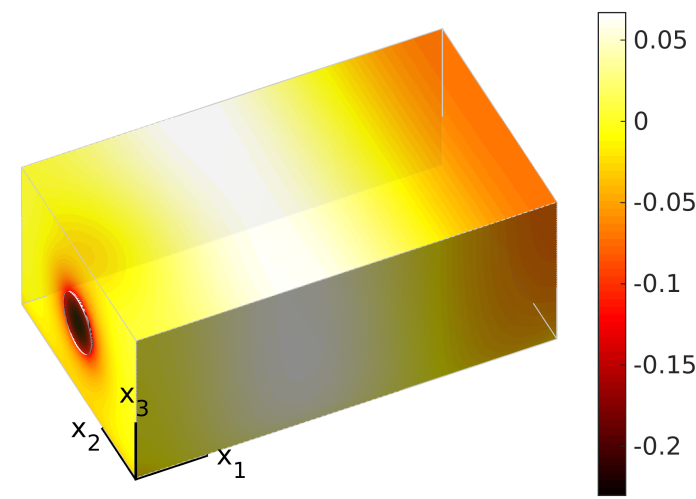

(a) $z_{N, M}^{*}\left[\mathcal{C}_{\tilde{k}}\right]$

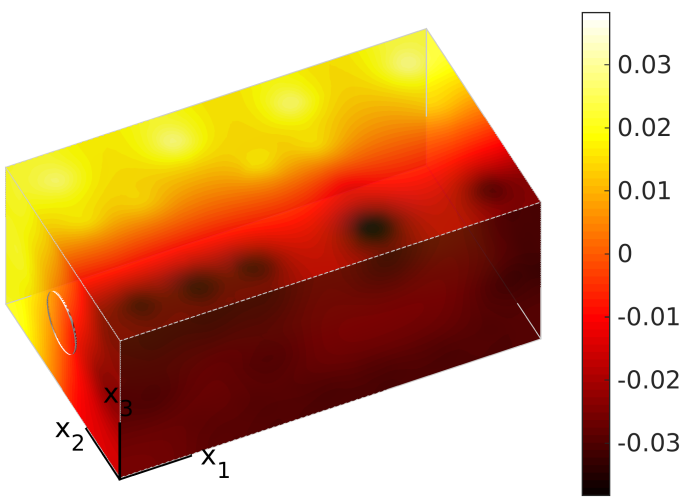

(b) $\eta_{N, M}^{*}\left[\mathcal{C}_{\tilde{k}}\right]$

Figure 11. The (a) background and (b) update component of the $N=7, M=48$ PBDW estimate to the $x_{2}$-antisymmetric resonance configuration.

\subsection{Data-Driven Empirical Enrichment of the Background Space}

We now devise a strategy to systematically incorporate the unmodeled physics identified by the update space $\mathcal{U}_{M}$ to augment the background space $\mathcal{Z}_{N}$ for subsequent data assimilation. The goal is to reduce the number of observations for future configurations. We consider the following algorithm:

(1) Find the configuration that maximizes the relative error (indicator):

$$
\tilde{k}^{*}=\underset{\tilde{k} \in[0.3,0.7]}{\arg \sup } \frac{E_{\text {avg }}\left[\mathcal{C}_{\tilde{k}}\right]\left(u_{N=N_{\max }, M=12}\right)}{E_{\text {avg }}\left[\mathcal{C}_{\tilde{k}}\right]\left(u_{N=0, M=0}\right)} .
$$

(2) Compute the update state associated with the configuration $\mathcal{C}_{\tilde{k}^{*}}$,

$$
\eta_{N=N_{\max }=8, M=M_{\max }=48}\left[\mathcal{C}_{\tilde{k}^{*}}\right] .
$$

(3) Construct the "augmented" best-knowledge space

$$
\begin{gathered}
\mathcal{Z}_{N_{\max }+1}^{\text {aug }} \equiv \operatorname{span}\left\{\mathcal{Z}_{N_{\max }}, \eta_{N=N_{\max }=8, M=M_{\max }=48}^{*}\left[\mathcal{C}_{\tilde{k}^{*}}\right]\right\} ; \\
\text { note that } \eta_{N_{\max }, M_{\max }}^{*}\left[\mathcal{C}_{\tilde{k}^{*}}\right] \in \mathcal{Z}_{N_{\max }}^{\perp} \cap \mathcal{U}_{M_{\max }} \text { and hence } \eta_{N_{\max }, M_{\max }}^{*}\left[\mathcal{C}_{\tilde{k}^{*}}\right] \text { is orthogonal to } \mathcal{Z}_{N_{\max }} .
\end{gathered}
$$

We then construct the PBDW system for $\mathcal{Z}_{N}^{\text {aug }}, N=1, \ldots, N_{\max }+1$. We wish to significantly reduce the background best-fit error through the incorporation of the unmodeled physics,

$$
\epsilon_{N_{\max }+1}^{\mathrm{bk}} \equiv \inf _{z \in \mathcal{Z}_{N_{\max }+1}^{\text {ug }}}\left\|u^{\text {true }}\left[\mathcal{C}_{\tilde{k}}\right]-z\right\| \ll \inf _{z \in \mathcal{Z}_{N_{\max }}}\left\|u^{\text {true }}\left[\mathcal{C}_{\tilde{k}}\right]-z\right\| \equiv \epsilon_{N_{\max }^{\mathrm{bk}}}
$$

for not only the configuration $\mathcal{C}_{\tilde{k}^{*}}$ but also for other configurations $\mathcal{C}_{\tilde{k}}$ of interest. In this way, we can reduce the required number of observations for future configurations.

We note that a reasonable approximation of the unmodeled physics, the antisymmetric mode, could require a large number of observations due to the rather slow convergence with $M$. Hence Step 2 can be experimentally expensive; however, we emphasize that the large number of observations is required only for the single configuration $\mathcal{C}_{\tilde{k}^{*}}$ with the (presumably) largest modeling error, and not for all the configurations of interest. Hence, in the many-query scenario in which we wish to deduce the state for many different configurations, the large experimental cost associated with the computation of the $\eta_{N_{\max }, M_{\max }}^{*}\left[\mathcal{C}_{\tilde{k}^{*}}\right]$ can be justified if the augmentation of the background space significantly reduces the best-knowledge error $\epsilon_{N_{\max }+1}^{\mathrm{bk}}$ for other configurations of interest. We note this is in fact the reduced-basis model-reduction philosophy [18]: we accept the large computational/experimental cost associated with select few configurations 


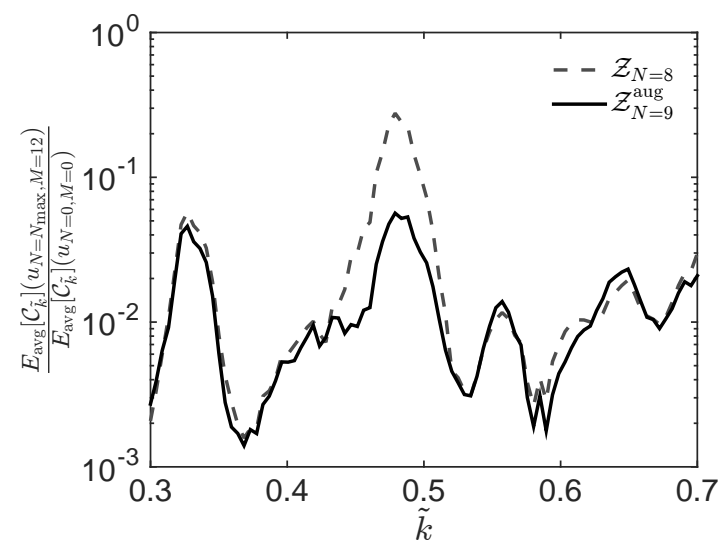

Figure 12. The relative error in the PBDW estimate for the original background space $\mathcal{Z}_{N=8}$ and the augmented background space $\mathcal{Z}_{N=9}^{\text {aug }}$ both using $M=12$ observations.

in the offline stage in order to significantly reduce the marginal cost associated with the solution of the computational/data-assimilation system for arbitrary configurations in the online stage.

We show in Figure 12 the relative error in the PBDW estimate for the original background space $\mathcal{Z}_{N=8}$ and the augmented background space $\mathcal{Z}_{N=9}^{\text {aug }}$. For the original background space, the maximum relative error is observed for the configuration $\mathcal{C}_{\tilde{k}^{*}=0.479}$ - precisely the configuration associated with the $x_{2}$-antisymmetric resonance. We hence augment the background space with the update field for $\tilde{k}^{*}=0.479$ associated with $M=48$ observations. The update field for $N=N_{\max }=8, M=48$ is similar to the update field for $N=7$, $M=48$ shown in Figure 11(b).

We observe in Figure 12 that the error for the PBDW estimate using the augmented background space $\mathcal{Z}_{N}^{\text {aug }}$ is considerably reduced compared to the error for the PBDW estimate using the original background space $\mathcal{Z}_{N}$, especially near the antisymmetric resonance at $\tilde{k}^{*}=0.479$. Note that the augmentation benefits not only the particular configuration $\tilde{k}^{*}=0.479$ for which the unmodeled physics is computed, but also other configurations in the vicinity of $\tilde{k}^{*}$. Hence, we effectively trade the high experimental cost associated with the estimation of $\eta_{N_{\max }, M_{\max }}^{*}\left[\mathcal{C}_{\tilde{k}^{*}}\right]$ for the reduction of the experimental cost associated with subsequent configurations. We show in Figure 13(a) the convergence of the PBDW estimate for $\tilde{k}^{*}=0.479$ as a function of the background space dimension $N$ and the number of observations $M$. We observe that, for $\mathcal{Z}_{N=9}^{\text {aug }}$ which includes the (approximation of the) $x_{2}$-antisymmetric mode, the error is small even for small $M(\geq 9)$. Figure 13(b) shows that, thanks to the inclusion of the $x_{2}$-antisymmetric mode in the background space, essentially all the energy is in the background component $z_{N=9, M}^{*}\left[\mathcal{C}_{\tilde{k}}\right]$.

In this particular example, we considered a single-step augmentation of the deficient background space $\mathcal{Z}_{N=8}$ by the antisymmetric mode. This single-step augmentation performs well for our acoustics problem because the solution to the Helmholtz problem at any frequency can be well approximated by a limited number of resonance modes in the vicinity of that frequency. In our particular case, the WeakGreedy algorithm first constructs the background space $\mathcal{Z}_{N=8}$ that captures essentially all the modes required to well represent $x_{2}$-Symmetric solutions; the update $\eta_{N_{\max }, M_{\max }}^{*}\left[\mathcal{C}_{\tilde{k}^{*}}\right]$ then adds the one $x_{2}$-antisymmetric resonance mode which is absent in the original background space. In general, we would need to iterate the augmentation procedure in, say, a greedy manner until the desired decrease in the error is obtained.

\section{ACKNOWLEDGMENT}

We thank Tommaso Taddei of MIT for fruitful discussions. This work was supported by the Foundation Sciences Mathématiques de Paris, OSD/AFOSR/MURI Grant FA9550-09-1-0613, ONR Grant N00014-111-0713, and the MIT-Singapore International Design Center. 


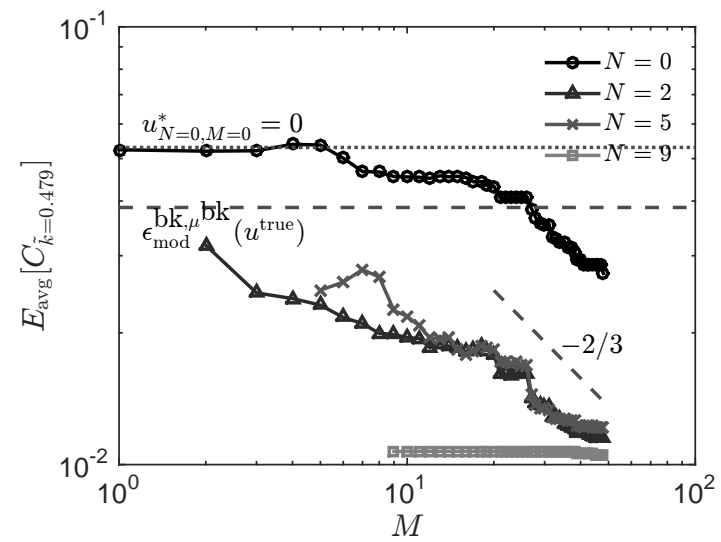

(a) $\tilde{k}=0.479$

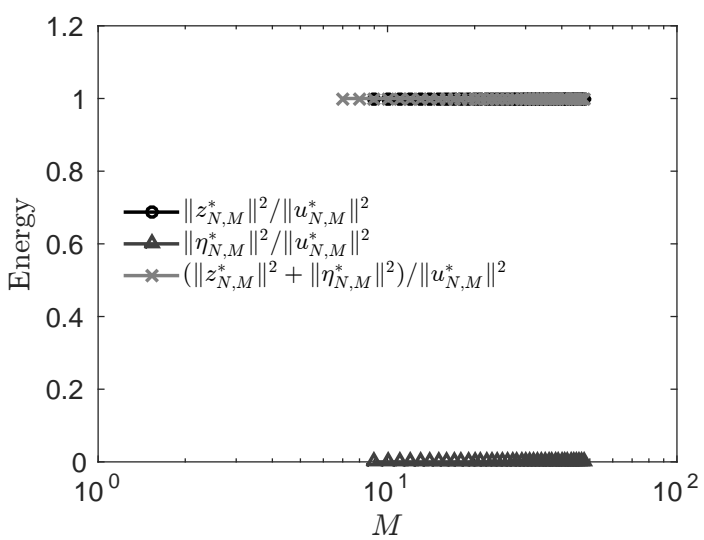

(b) energy fraction $(N=9)$

FiguRE 13. The convergence plot and the energy fraction decomposition for the $x_{2^{-}}$ symmetric resonance at $\tilde{k}=0.479$ using the augmented background space $\mathcal{Z}^{\text {aug }}$.

\section{REFERENCES}

[1] A. F. Bennett. Array design by inverse methods. Prog. Oceanog., 15:129-156, 1985.

[2] A. F. Bennett. Inverse Modeling of the Ocean and Atmosphere. Cambridge University Presss, Cambridge, 2002.

[3] P. Binev, A. Cohen, W. Dahmen, R. DeVore, G. Petrova, and P. Wojtaszczyk. Convergence rates for greedy algorithms in reduced basis methods. SIAM J. Math. Anal., 43:1457-1472, 2011.

[4] A. Buffa, Y. Maday, A. T. Patera, C. Prud'homme, and G. Turinici. A priori convergence of the greedy algorithm for the parametrized reduced basis method. Math. Model. Numer. Anal., 46:595-603, 2012.

[5] M. D. Buhmann. Radial basis functions: theory and implementations, volume 12. Cambridge university press, 2003.

[6] G. Chardon, A. Cohen, and L. Daudet. Sampling and reconstruction of solutions to the Helmholtz equation. Sampling Theory in Signal and Image Processing, page submitted, 2013.

[7] A. Cohen, M. Davenport, and D. Leviatan. On the stability and accuracy of least-squares approximations. Fond. Comput. Math., 13:819-834, 2013.

[8] R. DeVore, G. Petrova, and P. Wojtaszczyk. Greedy algorithms for reduced basis in Banach spaces. Constructive Approximation, 37:455-466, 2013.

[9] R. Everson and L. Sirovich. Karhunen-Loève procedure for gappy data. J. Opt. Soc. Am. A, 12(8):1657-1664, 1995.

[10] G. Franceschini and S. Macchietto. Model-based design of experiments for parameter precision: state of the art. Chem. Eng. Sci., 63:4846-4872, 2008.

[11] R. E. Kalman. A new approach to linear filtering and prediction problems. Transactions of the ASME-Journal of Basic Engineering, 82(Series D):35-45, 1960.

[12] Z. L. Li and I. M. Navon. Optimality of variational data assimilation and its relationship with the Kalman filter and smoother. Q.J.R. Meteorol, 127:661-683, 2001.

[13] A. C. Lorenc. A global three-dimensional multivariate statistical interpolation scheme. Mon. Wea. Rev., 109:701-721, 1981.

[14] Y. Maday and O. Mula. A generalized empirical interpolation method: application of reduced basis techniques to data assimilation. In F. Brezzi, P. C. Franzone, U. Gianazza, and G. Gilardi, editors, Analysis and numerics of partial differential equations, pages 221-235. Springer-Verlag, 2013.

[15] Y. Maday, O. Mula, A. T. Patera, and M. Yano. The generalized Empirical Interpolation Method: stability theory on Hilbert spaces with an application to the Stokes equation. Comput. Methods Appl. Mech. Engrg., in preparation.

[16] Y. Maday, A. T. Patera, J. D. Penn, and M. Yano. A parametrized-background data-weak approach to variational data assimilation: formulation, analysis, and application to acoustics. Internat. J. Numer. Methods Engrg., 2014.

[17] A. Quarteroni and A. Valli. Numerical Approximation of Partial Differential Equations. Springer, New York, 1997.

[18] G. Rozza, D. B. P. Huynh, and A. T. Patera. Reduced basis approximation and a posteriori error estimation for affinely parametrized elliptic coercive partial differential equations - application to transport and continuum mechanics. Archives of Computational Methods in Engineering, 15(3):229-275, 2008.

[19] T. Taddai and A. Patera. Experimental a posteriori error estimation by monte carlo sampling of observation functionals. Technical report, October 2014.

[20] K. Willcox. Unsteady flow sensing and estimation via the gappy proper orthogonal decomposition. Computers $\mathcal{E}$ Fluids, $35(2), 2006$.

[21] E. G. Williams. Fourier acoustics: sound radiation and near field acoustical holography. Academic Press, London, UK, 1999. 